\title{
THE THEORY OF $G^{\infty}$-SUPERMANIFOLDS
}

BY

\author{
CHARLES P. BOYER AND SAMUEL GITLER ${ }^{1}$
}

\begin{abstract}
A theory of supermanifolds is developed in which a supermanifold is an ordinary manifold associated with a certain integrable second order $G$-structure. A structure theorem is proved showing that every $G^{\infty}$-supermanifold has a complete distributive lattice of foliations with flat affine leaves. Furthermore, an existence and uniqueness theorem for local flows of $G^{\infty}$ vector fields is proved.
\end{abstract}

Introduction. Recently, with inspiration coming from physics there has been much interest in developing a theory of "supermanifolds". Such objects enable one to treat commutative and anticommutative variables on an equal footing. There have been essentially two approaches to this subject which can be qualified, loosely speaking, as the algebraic geometry approach and the differential geometry approach. The first approach has been developed by Berezin, Bernstein, Leites and coworkers [3-5, 11] in the Soviet Union and by Kostant [10]. This approach defines supermanifolds or graded manifolds by extending the (commutative) sheaf of functions on a manifold to a sheaf of $Z_{2}$-graded commutative algebras.

The second approach is due to DeWitt [8], Batchelor [1, 2], and to Rogers [15]. This approach mimics the usual definition of a manifold by replacing Euclidean space by supereuclidean space-the product of $m$-copies of the even part of an exterior algebra with $n$-copies of the odd part. Batchelor [2] shows that a categorical equivalence holds between these two approaches provided a coarse topology is used on supereuclidean space and that the "right" sheaf of functions is used. Moreover, Batchelor [1] shows that the isomorphism classes of "graded manifolds" defined by Kostant are in bijective correspondence with the sheaf of germs of sections of an associated exterior bundle over some vector bundle. However, this correspondence is not canonical. Thus a vector bundle imposes more structure.

The methods developed by Rogers [15] seem better suited to the needs of physicists. First, supereuclidean space is given a finer topology-that of a Banach

Received by the editors July 9, 1982 and, in revised form, July 5, 1983. Parts of the results presented here were announced at the Ninth International Colloquium on Group Theoretical Methods in Physics, Cocoyoc, Mexico in June 1980, and at the Symposium on Algebraic Topology in Honor of Jose Adem, Oaxtepec Mexico, in August 1981.

1980 Mathematics Subject Classification. Primary 58H05, 35G05; Secondary 83E99.

Key words and phrases. Almost supermanifolds, exterior algebra, foliations, pseudogroups, supereuclidean space, supermanifolds.

${ }^{1}$ This research was partially supported by the Consejo Nacional de Ciencia y Tecnologia under Grant PCCBBNA-005171. 
space. Secondly, a different sheaf of functions arises. Indeed, in the previous approaches, the only anticommuting elements at one's disposal are the generators of the exterior algebra which appear as "odd coordinates" of the manifold. Thus in this approach one does not treat explicitly the type of supersymmetry transformations which arise in physics. What is needed are constants with values in an exterior algebra. It is precisely this extra ingredient which arises in the sheaf of functions used by Rogers. However, a price must be paid. The "odd derivatives" are now not unique. This leads to several technical complications.

It is the purpose of the present paper to develop the theory of $G^{\infty}$-supermanifolds as begun by Rogers [15]. The outline of our paper is as follows: In $\$ 1$ we discuss differential calculus on supereuclidean spaces starting from Rogers' definition of derivative. The main result here is a theorem which completely characterizes $G^{\infty}$. functions as the solution space of a certain system of partial differential equations. The point being that a "supermanifold" will be an ordinary manifold with an added structure as in complex manifold theory.

In $\$ 2$ we discuss the super analog of the general linear group and determine its homotopy type. In the third section we describe the relevant pseudogroup of local $G^{\infty}$-diffeomorphisms. The technical point here being that this is a second order pseudogroup. We then use this pseudogroup to give the definition of a supermanifold in $\$ 4$. Actually there are two types of supermanifolds $-G^{1}$ - and $G^{\infty}$-supermanifolds.

In $\$ 5$ we give the definition of an almost supermanifold and give some nontrivial examples. We show that an almost supermanifold naturally determines two vector bundles over the manifold. In $\S 6$ we describe the tangent and supertangent bundles of a $G^{\infty}$-supermanifold. Here we give the super analog of the theory of local flows on a manifold. Finally, in $\$ 7$ we show that there is a certain multifoliate structure [9] associated with a supermanifold. The main theorem is that a $G^{\infty}$-supermanifold has a lattice of foliations all of whose leaves are flat affine manifolds.

A sequel to the present paper will devote itself to the theory of $G^{\infty}$ Lie groups and their homogeneous spaces. We also intend to address ourselves to probably the most important outstanding problem of this approach-Berezin integration [11]. We mention that most of the results proved in this paper were announced previously by the authors in [6, 7]. Finally, the authors would like to thank A. Haefliger, B. Kostant, S. Sternberg, and A. Verjovsky for discussions concerning this work.

The present paper is a revised version of an earlier manuscript. While making various changes suggested by the referee, we found that it proved much more convenient to generalize slightly the relevant class of functions and their derivations. Instead of dealing with functions with values in an exterior algebra $\Lambda$ we allow our functions to have values in certain quotient algebras of $\Lambda$. This seemingly innocuous generalization not only provides better economy of thought but also simplifies many proofs. The key here is the pairing (1.1). We would like to thank the referee for the constructive criticism which led to what we believe a much improved version. The earlier version of this paper was completed in 1981 when both authors were on sabbatical leave. The first author at Harvard University and the second at Brandeis 
University and we would like to thank the respective institutions for their hospitality.

1. Elementary analysis in supereuclidean space. Let $R^{N}$ be a euclidean $N$-space and $\Lambda=\Lambda\left(R^{N}\right)$ the exterior algebra on $R^{N}$. Then $\Lambda=\Lambda^{0}+\cdots+\Lambda^{N}$. Let $I_{k}=\Lambda^{k}+$ $\cdots+\Lambda^{N}$, thus $I_{0}=\Lambda, I_{N+1}=0$ and $I_{k}$ is an ideal in $\Lambda$. We denote by $\Lambda^{(k)}$ the quotient algebra of $\Lambda$ by $I_{k}$. There are natural pairings

$$
\phi_{l, k}: I_{l} \otimes \Lambda^{(k)} \rightarrow \Lambda^{(k+l)}
$$

induced by the pairings $I_{l} \otimes I_{k} \rightarrow I_{k+l}$. We have a sequence of epimorphisms

$$
\Lambda=\Lambda^{(N+1)} \rightarrow \Lambda^{(N)} \rightarrow \cdots \rightarrow \Lambda^{(1)} \rightarrow \Lambda^{(0)}=0
$$

which we call a cofiltration of $\Lambda$. Notice that if $k+l \leqslant N+1$, then $\phi_{l, k}(a \otimes x)=0$ for all $a$ and fixed $x$ implies $x=0$. We denote $\phi_{l, k}(a \otimes x)$ by $a * x$.

We give $\Lambda^{(k)}$ a $Z_{2}$-grading as follows. As a vector space,

$$
\Lambda^{(k)} \simeq \sum_{i \leqslant k-1} \Lambda^{i}
$$

Then set

$$
\Lambda_{0}^{(k)}=\sum_{2 i \leqslant k-1} \Lambda^{2 i}, \quad \Lambda_{1}^{(k)}=\sum_{2 i+1 \leqslant k-1} \Lambda^{2 i+1} .
$$

If we give $R^{N}$ the standard topology, then all the algebras $\Lambda^{(k)}$ become topologized and we denote by \|\| the norm.

Let $E_{N}^{m, n}=\left(\Lambda_{0} \times \cdots \times \Lambda_{0}\right) \times\left(\Lambda_{1} \times \cdots \times \Lambda_{1}\right)$ where there are $m$ copies of $\Lambda_{0}$ and $n$ copies of $\Lambda_{1}$ with the product topology. Then $E_{N}^{m, n}$ is homeomorphic to $R^{2^{N-1}(m+n)}$. Let $U \subset E_{N}^{m, n}$ be an open set. Then following A. Rogers [15], a function $f: U \rightarrow \Lambda^{(k)}$ is said to be of class $G^{1}$ (or $f \in G^{1}\left(U, \Lambda^{(k)}\right)$ ) if there exist continuous maps:

$$
G_{i} f: E_{N}^{m, n} \rightarrow \Lambda^{(k-|i|)}, \quad 1 \leqslant i \leqslant m+n,
$$

where $|i|=0$ if $1 \leqslant i \leqslant m,|i|=1$ if $m+1 \leqslant i \leqslant m+n$, and a function $\eta: E_{N}^{m, n} \rightarrow$ $\Lambda^{(k)}$ such that

$$
f(x+h)-f(x)=\sum_{i=1}^{m+n} h^{i} * G_{i} f(x)+\eta(h)\|h\|
$$

where $h=\left(h^{1}, \ldots, h^{m+n}\right)$ and $x \in E^{m, n},\|\eta\| \rightarrow 0$ as $\|h\| \rightarrow 0$ and we use the pairing (1.1) with $h^{i} \in I_{|i|}$. We call the $G_{i} f$ for $|i|=0$ the even derivatives of $f$ and the $G_{i} f$ for $|i|=1$ the odd derivatives of $f$. Thus the even derivatives of $f$ have the same cofiltration degree as $f$, while the odd derivatives decrease by one the cofiltration degree of $f$.

Proposition (1.3). The $G_{i}$ are graded derivations, i.e. they satisfy:

(1) $G_{i}(f+g)=G_{i}(f)+G_{i}(g)$,

(2) $G_{i}(f g)=\left(G_{i} f\right) g+(-1)^{|i||f|} f\left(G_{i} g\right)$

for $f, g \in G^{1}\left(U, \Lambda^{(k)}\right)$ and $f$ homogeneous, with $|f|$ its degree.

The definition of $G^{p}\left(U, \Lambda^{(k)}\right)$ is by induction. Namely $f$ is of class $G^{p}$ if the $G_{i} f$ are of class $G^{p-1}$. Note that if $f: E^{m, n} \rightarrow \Lambda^{(k)}$ is of class $G^{r}$ with $r \geqslant k$, then $G_{i_{1}} G_{i_{2}} \cdots G_{i_{r}} f=0$ if all the $\left|i_{j}\right|=1$ for $j=1, \ldots, r$. 
The result of Rogers in [15, Proposition 2.8] essentially gives

Proposition (1.4). Let $f \in G^{p}\left(U, \Lambda^{(k)}\right)$; then $f \in C^{p}\left(U, \Lambda^{(k)}\right)$. Moreover, regarded as a p-linear continuous map, the p-total derivative $D^{p} f$ is given by

$$
\left(D^{p} f(x)\right)\left(l_{1}, \ldots, l_{p}\right)=\sum_{k_{1}, \ldots, k_{p}=1}^{m+n}\left(l_{1}^{k_{1}} \cdots l_{p}^{k_{p}}\right) *\left(G_{k_{p}} G_{k_{p-1}} \cdots G_{k_{1}} f\right)(x)
$$

where $l_{i}=\left(l_{i}^{1}, \ldots, l_{i}^{m+n}\right)$ and $l_{1}^{k_{1}} \cdots l_{p}^{k_{p}}$ is the product in $\Lambda$.

Note that the pairing * makes sense, for if $\left(G_{k_{p}} \cdots G_{k_{1}}\right) f(x)$ decreases cofiltration by $s$, then $l_{1}^{k_{1}} \cdots l_{p}^{k_{p}}$ lies in $I_{s}$.

Let $e^{1}, \ldots, e^{N}$ be a basis for $R^{N}$. Then a basis for $\Lambda$ consists of $e^{\mu}$, where $\mu=\left(\mu_{1}, \ldots, \mu_{k}\right)$ is a sequence with $1 \leqslant \mu_{1}<\mu_{2}<\cdots<\mu_{k} \leqslant N$, where $e^{\mu}=e^{\mu_{1}}$ $\wedge \cdots \wedge e^{\mu_{k}}$ and $1=e^{0}$, where 0 corresponds to the empty sequence.

We denote by $\left\{e^{\mu}\right\}$ the induced basis also for $\Lambda^{(k)}$. If $f: U \rightarrow \Lambda^{(k)}$ we denote by $\partial f / \partial x_{\mu}^{i}: U \rightarrow \Lambda^{(k)}$ the partial derivative of $f$ with respect to the variable $x_{\mu}^{i}$ in the real subspace generated by $e^{\mu}$ considered in the $i$ th component of a vector in $E_{N}^{m, n}$, i.e.

$$
\frac{\partial f}{\partial x_{\mu}^{i}}(x)=\lim _{h \rightarrow 0} \frac{f\left(x+h\left(e^{\mu}\right)^{i}\right)-f(x)}{h} .
$$

Note that whenever we write $x_{\mu}^{i}$, then length of $\mu$ and $|i|$ have the same parity.

If $\nu \subset \mu$ are sequences, we let $\mu-\nu$ be the sequence obtained by deleting the elements of $\nu$ from $\mu$ and we let $\sigma(\mu, \nu)= \pm 1$ be defined by $e^{\mu}=\sigma(\mu, \nu) e^{\mu-\nu} e^{\nu}$.

From (1.4) one readily obtains

Proposition (1.5). A function $f \in C^{1}\left(U, \Lambda^{(k)}\right)$ is of class $G^{1}$ if and only if there exist continuous functions

$$
g_{i}(f): U \rightarrow \Lambda^{(k-|i|)}, \quad 1 \leqslant i \leqslant m+n,
$$

such that

$$
\partial f / \partial x_{\mu}^{i}=e^{\mu} * g_{i}(f), \quad 1 \leqslant i \leqslant m+n,
$$

for all $\mu$ with length of $\mu$ and $|i|$ of the same parity.

Moreover, the functions $g_{i}(f)$ are unique and in the definition of class $G^{1}$, we have $G_{i} f=g_{i}(f)$.

One of the main objectives of this section is to obtain the next theorem, which is a characterization of functions of class $G^{1}$ in terms of partial derivatives.

THEOREM (1.6). Let $U \subset E_{N}^{m, n}$ be open and $f \in C^{1}\left(U, \Lambda^{(k)}\right)$. Then $f \in G^{1}\left(U, \Lambda^{(k)}\right)$ if and only if the following relations hold for the partial derivatives:

(i) $\partial f / \partial x_{\mu}^{i}=\sigma(\mu, \nu) e^{\mu-\nu} \partial f / \partial x_{\nu}^{i}$ if $\nu \subset \mu$ and $\mu-\nu$ has even length.

(ii) $e^{a} * \partial f / \partial x_{b}^{i}+e^{b} * \partial f / \partial x_{a}^{i}=0$ in $C^{1}\left(U, \Lambda^{(k+1)}\right)$ for $|i|=1$ and $a, b=1, \ldots, N$. 
Proof. Suppose $f \in G^{1}\left(U, \Lambda^{(k)}\right)$, then by (1.5) we have $\partial f / \partial x_{\mu}^{i}=e^{\mu} * G_{i} f$; therefore if $\nu \subset \mu$ and both have lengths of the same parity, i.e. $\mu-\nu$ has even length,

$$
\frac{\partial f}{\partial x_{\mu}^{i}}=\sigma(\mu, \nu) e^{\mu-\nu} e^{\nu} * G_{i} f=\sigma(\mu, \nu) e^{\mu-\nu} \frac{\partial f}{\partial x_{\nu}^{i}}
$$

so that (i) holds.

Now again from (1.5), we have for $a, b=1, \ldots, N$ and $|i|=1$,

$$
e^{a} * \frac{\partial f}{\partial x_{b}^{i}}+e^{b} * \frac{\partial f}{\partial x_{a}^{i}}=e^{a} * e^{b} * G_{i} f+e^{b} * e^{a} * G_{i} f=0
$$

so that (ii) holds. Thus the necessity of the conditions (i) and (ii).

Conversely, to show the sufficiency, by (1.5) it suffices to construct the functions $g_{i}(f)$. For $|i|=0$, i.e. $i=1, \ldots, m$, set $g_{i}(f)=\partial f / \partial x_{0}^{i}$, then for every sequence $\mu$ of even length, using (i) we have

$$
\frac{\partial f}{\partial x_{\mu}^{i}}=e^{\mu} \frac{\partial f}{\partial x_{0}^{i}}=e^{\mu} * g_{i}(f) .
$$

To finish the proof, we have to construct functions

$$
g_{i}(f): U \rightarrow \Lambda^{(k-1)}, \quad m+1 \leqslant i \leqslant m+n,
$$

satisfying $\partial f / \partial x_{\mu}^{i}=e^{\mu} * g_{i}(f)$ for all sequences $\mu$ of odd length.

We use an algebraic lemma that was kindly suggested by the referee.

LemMA (1.7). If $A$ is any algebra and $I_{1}, \ldots, I_{n}$ are ideals in $A$, there is an exact sequence

$$
\bigcap_{k} I_{k} \stackrel{i}{\rightarrow} A \stackrel{\alpha}{\rightarrow} \bigoplus_{k} A / I_{k} \stackrel{\beta}{\rightarrow} \underset{(k, j)}{\bigoplus} A /\left(I_{k}+I_{j}\right)
$$

where $i$ is the inclusion, $\alpha$ is the diagonal followed by the natural projection, $(k, j)$ runs over all pairs $n \geqslant k>j \geqslant 1$ and

$$
\beta\left(\left[a_{1}\right], \ldots,\left[a_{n}\right]\right)=\left(\left[a_{2}-a_{1}\right],\left[a_{3}-a_{1}\right],\left[a_{3}-a_{2}\right], \ldots,\left[a_{n}-a_{n-1}\right]\right) .
$$

The proof of (1.7) follows easily by induction on $n$.

Fix $i$, and let $\partial f / \partial x_{\mu}^{i}=f_{\mu}$. The conditions (i) and (ii) can be rewritten as:

(i) $f_{\mu}=\sigma(\mu, \nu) e^{\mu-\nu} f_{\nu}$ if $\nu \subset \mu$ and $\nu$ and $\mu$ have odd length.

(ii) $e^{a} * f_{b}+e^{b} * f_{a}=0$ in $C^{r}\left(U, \Lambda^{(k+1)}\right)$ for $a, b=1, \ldots, N$.

By (ii)' when $a=b$, we have $2 e^{a} * f_{a}=0$ but then $f_{a}$ is in the principal ideal generated by $e^{a}$ in $C^{r}\left(U, \Lambda^{(k)}\right)$, and which we denote by $I^{(k)}\left(e^{a}\right)$. Thus $f_{a}=e^{a} g_{a}$ for some function $g_{a} \in C^{r}\left(U, \Lambda^{(k)}\right)$. If $\bar{g}_{a}$ is the projection of $g_{a}$ to $C^{r}\left(U, \Lambda^{(k-1)}\right)$, then $\bar{g}_{a}$ is unique modulo $I^{(k-1)}\left(e^{a}\right)$. We now apply Lemma (1.7) with $A=C^{r}\left(U, \Lambda^{(k-1)}\right)$ and ideals $I^{(k-1)}\left(e^{1}\right), \ldots, I^{(k-1)}\left(e^{N}\right)$.

Consider the element $\left(\left[g_{1}\right], \ldots,\left[g_{N}\right]\right) \in \oplus_{j} C^{r} /\left(U, \Lambda^{(k-1)}\right) / I^{(k-1)}\left(e^{j}\right)$. We claim $\beta\left(\left[g_{1}\right], \ldots,\left[g_{N}\right]\right)=0$. We have

$$
f_{a}=e^{a} g_{a}=e^{a} * \bar{g}_{a}
$$


and from (ii)', we obtain

$$
e^{a} * e^{b} * \bar{g}_{b}+e^{b} * e^{a} * \bar{g}_{a}=0 \quad \text { in } C^{r}\left(U, \Lambda^{(k+1)}\right)
$$

thus

$$
\bar{g}_{a}=\bar{g}_{b} \bmod \left(I^{(k-1)}\left(e^{a}\right)+I^{(k-1)}\left(e^{b}\right)\right)
$$

and hence $\beta\left(\left[g_{1}\right], \ldots,\left[g_{n}\right]\right)=0$.

Then by (1.7), there exists $g \in C^{r}\left(U, \Lambda^{(k-1)}\right)$ with $\alpha(g)=\left(\left[g_{1}\right], \ldots,\left[g_{n}\right]\right)$. Moreover, $\bigcap_{a=1}^{N} I^{(k-1)}\left(e^{a}\right)=0$ so that $g$ is unique. By definition,

$$
g=\bar{g}_{a} \bmod I^{(k-1)}\left(e^{a}\right) .
$$

Hence

$$
f_{a}=e^{a} * \bar{g}_{a}=e^{a} * g .
$$

Since $\mu$ is a sequence of odd length say $\mu=\left(\mu_{1}, \ldots, \mu_{2 a+1}\right)$

$$
f_{\mu}=e^{\hat{\mu}} f_{2 a+1}=e^{\hat{\mu}} e^{2 a+1} * g=e^{\mu} * g
$$

where $\hat{\mu}=\left(\mu_{1}, \ldots, \mu_{2 a}\right)$, by (i) and the above. Thus $g=g_{i}(f)$ is unique in $C^{r}\left(U, \Lambda^{(k-1)}\right)$ and satisfies $\partial f / \partial x_{\mu}^{i}=e^{\mu} * g_{i}(f)$ for all sequences $\mu$ of odd length. This finishes the proof of (1.6).

REMARK (1.8). Equations (i) and (ii) of (1.6) are the analogues of the CauchyRiemann equations.

Corollary (1.9). If $f \in C^{2}\left(U, \Lambda^{(k)}\right) \cap G^{1}\left(U, \Lambda^{(k)}\right)$, then the even derivatives $G_{i} f$ are also in $G^{1}\left(U, \Lambda^{(k)}\right)$.

Proof. Because of (1.6), $G_{i} f=\partial f / \partial x_{0}^{i}, 1 \leqslant i \leqslant m$. Hence

$$
\begin{aligned}
\frac{\partial}{\partial x_{\mu}^{j}}\left(G_{i} f\right) & =\frac{\partial}{\partial x_{\mu}^{j}} \frac{\partial f}{\partial x_{0}^{i}}=\frac{\partial}{\partial x_{0}^{i}} \frac{\partial f}{\partial x_{\mu}^{j}}=\frac{\partial}{\partial x_{0}^{i}}\left(e^{\mu} * G_{j} f\right) \\
& =e^{\mu} * \frac{\partial}{\partial x_{0}^{i}}\left(G_{j} f\right) .
\end{aligned}
$$

Hence if $\nu \subset \mu$ and $\mu-\nu$ has even length,

$$
\begin{aligned}
\frac{\partial}{\partial x_{\mu}^{j}}\left(G_{i} f\right) & =\sigma(\mu, \nu) e^{\mu-\nu} e^{\nu} \frac{\partial}{\partial x_{0}^{i}}\left(G_{j} f\right) \\
& =\sigma(\mu, \nu) e^{\mu-\nu} \frac{\partial}{\partial x_{\nu}^{j}}\left(G_{i} f\right)
\end{aligned}
$$

so that (i) of (1.6) holds for $G_{i} f$. Now consider

$$
\begin{aligned}
e^{a} * \frac{\partial}{\partial x_{b}^{j}}\left(G_{i} f\right) & =e^{a} * \frac{\partial}{\partial x_{b}^{j}} \frac{\partial f}{\partial x_{0}^{i}}=e^{a} * \frac{\partial}{\partial x_{0}^{i}} \frac{\partial f}{\partial x_{b}^{j}} \\
& =e^{a} * \frac{\partial}{\partial x_{0}^{i}}\left(e^{b} G_{j} f\right)=e^{a} *\left(e^{b} \frac{\partial}{\partial x_{0}^{i}} G_{j} f\right) .
\end{aligned}
$$

Also

$$
e^{b} * \frac{\partial}{\partial x_{a}^{j}}\left(G_{i} f\right)=e^{b} *\left(e^{a} \frac{\partial}{\partial x_{0}^{i}} G_{j} f\right)
$$

and hence (ii) of (1.6) holds for $G_{i} f,|i|=0$, and $G_{i} f$ is of class $G^{1}$. 
Now we have

Proposition (1.10). If $f \in G^{1}\left(U, \Lambda^{(k)}\right) \cap C^{2}\left(U, \Lambda^{(k)}\right)$, then
(a) $\frac{\partial f}{\partial x_{\mu}^{i}} \in G^{1}\left(U, \Lambda^{(k)}\right)$.
(b) $G_{j}\left(\frac{\partial f}{\partial x_{\mu}^{i}}\right)=\frac{\partial}{\partial x_{\mu}^{i}}\left(G_{j} f\right)$.
(c) $\frac{\partial^{2} f}{\partial x_{a}^{i} \partial x_{b}^{j}}+\frac{\partial^{2} f}{\partial x_{b}^{i} \partial x_{a}^{j}}=0$ in $\begin{cases}C\left(U, \Lambda^{(k)}\right) & \text { if } k \leqslant N, \\ C\left(U, \Lambda^{(N)}\right) & \text { if } k=N+1 .\end{cases}$

Proof. (a) We have $\partial f / \partial x_{\mu}^{i}=e^{\mu} * G_{i} f$. For $i \leqslant m$, the $G_{i} f \in G^{1}\left(U, \Lambda^{(k)}\right)$ by (1.9), hence so are the $\partial f / \partial x_{\mu}^{i}$, with $i \leqslant m$. Now for $i>m$, suppose $\nu \subset \rho$ and $\rho-\nu$ has even length, then

$$
\begin{aligned}
\frac{\partial}{\partial x_{\rho}^{j}}\left(\frac{\partial f}{\partial x_{\mu}^{i}}\right) & =\frac{\partial}{\partial x_{\mu}^{i}}\left(\frac{\partial f}{\partial x_{\rho}^{j}}\right)=\frac{\partial}{\partial x_{\mu}^{i}}\left(e^{\rho} * G_{j} f\right) \\
& =\sigma(\rho, \nu) e^{\rho-\nu} \frac{\partial}{\partial x_{\mu}^{i}}\left(e^{\nu} * G_{j} f\right) \\
& =\sigma(\rho, \nu) e^{\rho-\nu} \frac{\partial}{\partial x_{\mu}^{i}}\left(\frac{\partial f}{\partial x_{\nu}^{j}}\right) \\
& =\sigma(\mu, \nu) e^{\rho-\nu} \frac{\partial}{\partial x_{\nu}^{j}}\left(\frac{\partial f}{\partial x_{\mu}^{i}}\right)
\end{aligned}
$$

so that (i) of (1.6) holds.

Now consider

$$
\begin{aligned}
& e^{a} * \frac{\partial}{\partial x_{b}^{j}}\left(\frac{\partial f}{\partial x_{\mu}^{i}}\right)=e^{a} * \frac{\partial}{\partial x_{\mu}^{i}}\left(e^{b} * G_{j} f\right), \\
& e^{b} * \frac{\partial}{\partial x_{b}^{j}}\left(\frac{\partial f}{\partial x_{\mu}^{i}}\right)=e^{b} * \frac{\partial}{\partial x_{\mu}^{i}}\left(e^{a} * G_{j} f\right)
\end{aligned}
$$

and (ii) of (1.6) holds. Hence the proof of (a) is complete.

To prove (b) consider $j>m$ first, then

$$
\begin{aligned}
\frac{\partial}{\partial x_{\mu}^{i}}\left(f\left(x+h^{j}\right)-f(x)\right) & =\frac{\partial}{\partial x_{\mu}^{i}}\left(h^{j} * G_{j} f+\eta\left(h^{j}\right)\left\|h^{j}\right\|\right) \\
& =\frac{\partial f}{\partial x_{\mu}^{i}}\left(x+h^{j}\right)-\frac{\partial f}{\partial x_{\mu}^{i}}(x) \\
& =\left(h^{j} * G_{j}\left(\frac{\partial f}{\partial x_{\mu}^{i}}\right)+\eta^{\prime}\left(h^{j}\right)\left\|h^{j}\right\|\right)
\end{aligned}
$$


and (b) follows for $j>m$. For $j \leqslant m$, we have

$$
G_{j}\left(\frac{\partial f}{\partial x_{\mu}^{i}}\right)=\frac{\partial^{2} f}{\partial x_{0}^{j} \partial x_{\mu}^{i}}=\frac{\partial^{2} f}{\partial x_{\mu}^{i} \partial x_{0}^{j}}=\frac{\partial}{\partial x_{\mu}^{i}}\left(G_{j} f\right)
$$

and the proof of $(b)$ is complete.

Finally, for the proof of (c), consider,

$$
\begin{aligned}
\frac{\partial^{2} f}{\partial x_{a}^{i} \partial x_{b}^{j}} & =\frac{\partial}{\partial x_{a}^{i}}\left(e^{b} * G_{j} f\right)=e^{b} * \frac{\partial}{\partial x_{a}^{i}} G_{j} f \\
& =e^{b} * G_{j}\left(\frac{\partial f}{\partial x_{a}^{i}}\right)=-G_{j}\left(e^{b} * \frac{\partial f}{\partial x_{a}^{i}}\right) \\
& =G_{j}\left(e^{a} * \frac{\partial f}{\partial x_{b}^{i}}\right)=-e^{a} * G_{j}\left(\frac{\partial f}{\partial x_{b}^{i}}\right) \\
& =-\frac{\partial}{\partial x_{a}^{j}}\left(\frac{\partial f}{\partial x_{b}^{i}}\right)=-\frac{\partial^{2} f}{\partial x_{b}^{i} \partial x_{a}^{j}}
\end{aligned}
$$

and (c) follows. The difference for $k \leqslant N$ and $k>N$ appears that when $k \leqslant N$, $e^{b} * \partial f / \partial x_{a}^{i} \in C^{1}\left(U, \Lambda^{(k+1)}\right)$ and then $G_{j}$ drops it down to $C^{0}\left(U, \Lambda^{(k)}\right)$ while for $k=N+1, e^{b} * \partial f / \partial x_{a}^{i} \in C^{1}(U, \Lambda)$ and $G_{j}$ drops it to $C^{0}\left(U, \Lambda^{(N)}\right)$.

THEOREM (1.11). If $f \in G^{1}\left(U, \Lambda^{(k)}\right) \cap C^{\infty}\left(U, \Lambda^{(k)}\right)$ and $k \leqslant N$, then $f \in$ $G^{\infty}\left(U, \Lambda^{(k)}\right)$.

Proof. By (1.9), the $G_{j} f$ for $j \leqslant m$ are in $G^{1}\left(U, \Lambda^{(k)}\right) \cap C^{\infty}\left(U, \Lambda^{(k)}\right)$. We now prove conditions (i) and (ii) for $G_{j} f$, when $j>m$. If $\nu \subset \mu$ and $\mu-\nu$ has even length, we have

$$
\begin{aligned}
\frac{\partial}{\partial x_{\mu}^{i}}\left(G_{j} f\right) & =G_{j}\left(\frac{\partial f}{\partial x_{\mu}^{i}}\right)=G_{j}\left(e^{\mu} * G_{i} f\right) \\
& =\sigma(\mu, \nu) G_{j}\left(e^{\mu-\nu} * e^{\nu} * G_{i} f\right) \\
& =\sigma(\mu, \nu) e^{\mu-\nu} * G_{j}\left(e^{\nu} * G_{i} f\right) \\
& =\sigma(\mu, \nu) e^{\mu-\nu} * G_{j}\left(\frac{\partial}{\partial x_{\nu}^{i}} f\right) \\
& =\sigma(\mu, \nu) e^{\mu-\nu} * \frac{\partial}{\partial x_{\nu}^{i}}\left(G_{j} f\right)
\end{aligned}
$$

so that (i) holds in $C^{\infty}\left(U, \Lambda^{(k-1)}\right)$. Now consider

$$
\begin{aligned}
e^{a} * \frac{\partial}{\partial x_{b}^{i}}\left(G_{j} f\right) & =e^{a} * G_{j}\left(\frac{\partial f}{\partial x_{b}^{i}}\right)=-G_{j}\left(e^{a} * \frac{\partial f}{\partial x_{b}^{i}}\right) \\
& =G_{j}\left(e^{b} * \frac{\partial f}{\partial x_{a}^{i}}\right)=-e^{b} * G_{j}\left(\frac{\partial f}{\partial x_{a}^{i}}\right)=-e^{b} * \frac{\partial}{\partial x_{a}^{i}}\left(G_{j} f\right)
\end{aligned}
$$


and then (ii) of (1.6) holds for $G_{j} f$. Thus we have shown that if $f \in G^{1}\left(U, \Lambda^{(k)}\right) \cap$ $C^{\infty}\left(U, \Lambda^{(k)}\right)$, then $G_{i} f \in G^{1}\left(U, \Lambda^{(k-|i|)}\right) \cap C^{\infty}\left(U, \Lambda^{(k-|i|)}\right)$ if $k \leqslant N$. Hence we may proceed by induction.

THEOREM (1.12). If $f \in G^{1}(U, \Lambda) \cap C^{\infty}(U, \Lambda)$, then $f \in G^{\infty}(U, \Lambda)$ if and only if

$$
\frac{\partial^{2} f}{\partial x_{a}^{i} \partial x_{b}^{j}}+\frac{\partial^{2} f}{\partial x_{b}^{i} \partial x_{a}^{j}}=0
$$

Proof. First sufficiency. As in (1.11), it follows that the $G_{j} f$ are in $G^{1}(U, \Lambda) \cap$ $C^{\infty}(U, \Lambda)$ if $j \leqslant m$. Also as in (1.11), condition (i) of (1.6) holds for $G_{j} f$ if $j>m$. Now to prove

$$
e^{a} * \frac{\partial G_{j} f}{\partial x_{b}^{i}}+e^{b} * \frac{\partial G_{j} f}{\partial x_{a}^{i}}=0 \quad \text { in } C^{\infty}(U, \Lambda)
$$

we have

$$
\begin{aligned}
e^{a} * \frac{\partial}{\partial x_{b}^{i}} G_{j} f & =e^{a} * G_{j}\left(\frac{\partial f}{\partial x_{b}^{i}}\right)=\frac{\partial}{\partial x_{a}^{j}} \frac{\partial f}{\partial x_{b}^{i}}=\frac{\partial}{\partial x_{b}^{i}} \frac{\partial f}{\partial x_{a}^{j}} \\
& =-\frac{\partial^{2} f}{\partial x_{a}^{i} \partial x_{b}^{j}}=-\frac{\partial^{2} f}{\partial x_{b}^{j} \partial x_{a}^{i}}=-e^{b} * G_{j}\left(\frac{\partial f}{\partial x_{a}^{i}}\right) \\
& =-e^{b} * \frac{\partial}{\partial x_{a}^{i}}\left(G_{j} f\right)
\end{aligned}
$$

and hence under the hypothesis, we see that

$$
G_{j} f \in G^{1}\left(U, \Lambda^{(N+1-|j|)}\right) \cap C^{\infty}\left(U, \Lambda^{(N+1-|j|)}\right) .
$$

By (1.9) and (1.11), we may proceed by induction.

Finally to prove necessity: because

$$
e^{a} * \frac{\partial G_{j} f}{\partial x_{b}^{i}}+e^{b} * \frac{\partial G_{j} f}{\partial x_{a}^{i}}=0 \quad \text { in } C^{\infty}(U, \Lambda)
$$

we have, reinverting the argument above, that (1.13) holds.

We may summarize the previous results as

THeOREM (1.14). A function $f \in C^{\infty}\left(U, \Lambda^{(k)}\right)$ is in $G^{\infty}\left(U, \Lambda^{(k)}\right)$ for $k \leqslant N$ if and only if

(i) For any sequences $\mu \supset \nu$, with $\mu-\nu$ of even length

$$
\frac{\partial f}{\partial x_{\mu}^{i}}=\sigma(\mu, \nu) e^{\mu-\nu} \frac{\partial f}{\partial x_{\nu}^{i}} .
$$

(ii) $e^{a} * \partial f / \partial x_{b}^{i}+e^{b} * \partial f / \partial x_{a}^{i}=0$ in $C^{\infty}\left(U, \Lambda^{(k+1)}\right),|i|=1$ and $a, b=1, \ldots, N$.

Moreover for $k=N+1$, we need the extra condition

(iii) $\partial^{2} f / \partial x_{a}^{i} \partial x_{b}^{j}+\partial^{2} f / \partial x_{b}^{i} \partial x_{a}^{j}=0$ in $C^{\infty}(U, \Lambda)$ for all $|i|=|j|=1$ and $a, b=$ $1, \ldots, N$. 
For iterates of the $G_{i}$ we have

Proposition (1.15). Let $f \in G^{2}\left(U, \Lambda^{(k)}\right)$. Then

$$
G_{i} G_{j} f=(-1)^{|i||j|} G_{j} G_{i} f
$$

in $C^{0}\left(U, \Lambda^{(k-|i|-|j|)}\right)$.

We now recall several extension theorems due to A. Rogers in [15].

Consider $E^{m, n} \stackrel{\rho}{\rightarrow} E^{m, 0} \stackrel{\varepsilon_{0}}{\rightarrow} R^{m}$, where $\rho$ is the projection and $\varepsilon_{0}$ is the augumentation. We let $\eta_{0}: R^{m} \rightarrow E^{m, 0}$ and $\eta: R^{m} \rightarrow E^{m, n}$ be the "obvious" inclusions.

If $U \subset E^{m, n}$ is open, $\rho(U)=U_{0}$ and $\varepsilon(U)$ are open in $E^{m, 0}$ and $R^{m}$, respectively.

TheOREM (1.16). Let $\tilde{f} \in C^{\infty}\left(\varepsilon(U), \Lambda^{(k)}\right)$. Then there is a unique $f \in G^{\infty}\left(U_{0}, \Lambda^{(k)}\right)$ such that $f\left(\eta_{0}(x)\right)=\tilde{f}(x)$.

REMARK (1.17). Rogers calls such an $f$ a $z$-expansion of $\tilde{f}$. Explicitly,

$$
f(x)=z(\tilde{f})(x)=\sum_{i_{1} \cdots i_{m}} \frac{s\left(x^{1}\right)^{i_{1}} \cdots s\left(x^{m}\right)^{i_{m}}}{i_{1} ! \cdots i_{m} !} \partial_{i_{1}} \cdots \partial_{i_{m}} \tilde{f}\left(x_{0}\right)
$$

where $\partial_{i}=\partial / \partial x_{0}^{i}, x=\left(x^{1}, \ldots, x^{m}\right), x_{0}=\left(x_{0}^{1}, \ldots, x_{0}^{m}\right), \varepsilon_{0}(x)=x_{0}$, and $s(\lambda)=\lambda-$ $\varepsilon(\lambda) 1$, for $\lambda \in \Lambda$. Moreover for $i=1, \ldots, m$,

$$
G_{i} z(\tilde{f})=z\left(\partial \tilde{f} / \partial x_{0}^{i}\right)
$$

The result (1.16) generalizes to

THEOREM (1.18) Let $U \subset W \subset E^{m, n}$ be open sets with $\varepsilon(U)=\varepsilon(W)$ and let $f \in G^{\infty}\left(U, \Lambda^{(k)}\right)$. Then there exists a unique $f^{\prime} \in G^{\infty}\left(W, \Lambda^{(k)}\right)$, such that $f^{\prime} \mid U=f$.

REMARK (1.19). The result (1.18) is not true for $G^{1}$-functions.

As a corollary to (1.18) one has

TheOREM (1.20). Let $f \in C^{\infty}\left(U, \Lambda^{(k)}\right)$. Suppose $f \in G^{\infty}\left(U, \Lambda^{(k)}\right)$, then there exist unique functions $f_{\mu} \in C^{\infty}\left(\varepsilon(U), \Lambda^{(k)}\right)$, where $\mu$ runs through all sequences $\left(\mu_{1}, \ldots, \mu_{j}\right)$ with $1 \leqslant \mu_{1}<\mu_{2}<\cdots<\mu_{j} \leqslant n$, such that

$$
f(x, y)=\sum y^{\mu} * z\left(\tilde{f}_{\mu}\right)(x) \text {. }
$$

Conversely suppose given $\left\{\tilde{f}_{\mu}\right\} \in C^{\infty}\left(\varepsilon(U), \Lambda^{(k)}\right)$, then $f$ defined by (1.21) is in $G^{\infty}\left(U, \Lambda^{(k)}\right)$.

REMARK (1.22). The functions $\tilde{f}_{\mu}$ are obtained as follows: Choose $W=\varepsilon^{-1}(\varepsilon(U))$ in $E^{m, n}$, and let $f^{\prime}$ be the unique extension of $f$ to $W$. Define $\rho: G^{\infty}\left(U, \Lambda^{(k)}\right) \rightarrow$ $C^{\infty}\left(\varepsilon(U), \Lambda^{(k)}\right)$ by $\rho(g)\left(x_{1}, \ldots, x_{m}\right)=g^{\prime}\left(x_{1} 1, \ldots, x_{m} 1\right)$. Then let

$$
\tilde{f}_{0}=\rho\left(f^{\prime}\right), \quad \tilde{f}_{\mu}=\rho\left(G_{\mu_{1}+m} \cdots G_{\mu_{j}+m} f^{\prime}\right) .
$$

Theorem (1.20) allows us to describe the algebra $G^{\infty}\left(U, \Lambda^{(k)}\right)$ as follows. Let $V$ be an $n$-dimensional real vector space, then 
THEOREM (1.23). There is an algebra isomorphism induced by the z-expansion of Rogers,

$$
G^{\infty}\left(U, \Lambda^{(k)}\right) \simeq \sum_{p=0}^{\min (k, n)} \Lambda^{p}(V) \otimes C^{\infty}\left(\varepsilon(U), \Lambda^{(k-p)}\right)
$$

where the right-hand side has the natural graded algebra structure and $V$ is the vector space generated by the odd coordinates $y^{1}, \ldots, y^{n}$.

2. The linear groups. Suppose $f: E^{m, n} \rightarrow E^{r, s}$ is a map. Let $\Pi^{j}: E^{r, s} \rightarrow \Lambda_{|j|}$ be the projection and denote by $f^{j}$ the composition $\Pi^{j} f$. We say $f$ is $G^{1}\left(G^{\infty}\right)$ if each of the $\operatorname{maps} f^{j}$ is $G^{1}\left(G^{\infty}\right)$.

If $f: E^{m, n} \rightarrow E^{m, n}$ is $G^{1}$, then we may form an $(m+n) \times(m+n)$ matrix

$$
G_{i} f^{j}(x) \text { for } x \in E^{m, n}
$$

where $G_{i} f^{j}(x) \in \Lambda_{|i|+|j|}^{(N+1-|i|)}$ and $|i|+|j|$ is taken mod 2. Explicitly,

$$
\begin{aligned}
& G_{i} f^{j}(x) \in \Lambda_{0} \quad \text { if } 1 \leqslant i \leqslant m \text { and } 1 \leqslant j \leqslant m, \\
& G_{i} f^{j}(x) \in \Lambda_{1} \quad \text { if } 1 \leqslant i \leqslant m \text { and } m+1 \leqslant j \leqslant m+n, \\
& G_{i} f^{j}(x) \in \Lambda_{1}^{(N)} \quad \text { if } m+1 \leqslant i \leqslant m+n \text { and } 1 \leqslant j \leqslant m, \\
& G_{i} f^{j}(x) \in \Lambda_{0}^{(N)} \quad \text { if } m+1 \leqslant i \leqslant m+n \text { and } m+1 \leqslant j \leqslant m+n .
\end{aligned}
$$

More generally, consider $(m+n) \times(m+n)$ square matrices

$$
\bar{A}=\left(\begin{array}{ll}
a & \bar{\xi} \\
\eta & \bar{b}
\end{array}\right)
$$

where the entries of $a$ lie in $\Lambda_{0}$ and $a$ is an $m \times m$ matrix, $\eta$ is an $n \times m$ matrix with entries in $\Lambda_{1}, \bar{\xi}$ is an $m \times n$ matrix with entries in $\Lambda_{1}^{(N)}$ and $\bar{b}$ is an $n \times n$ matrix with entries in $\Lambda_{0}^{(N)}$.

We may compose matrices like $\bar{A}$ using the pairing (1.1) when necessary. We say the matrix $\bar{A}$ is invertible if and only if there exists a matrix $\bar{B}$ so that $\bar{A} \bar{B}=\bar{I}$, where $\bar{I}=\left(\begin{array}{ll}I & 0 \\ 0 & I\end{array}\right)$. We denote by $G_{N}(m \mid n)$ the group of invertible matrices thus formed.

There is another group $G L_{N}(m \mid n)$ that has been studied in [14], and is obtained as follows. Consider the $(m+n) \times(m+n)$ matrices $A=\left(\begin{array}{c}a \xi \\ \eta b\end{array}\right)$ where $a$ and $\eta$ are as in the matrix $\bar{A}$, while $\xi$ and $b$ have entries in $\Lambda_{1}$ and $\Lambda_{0}$ respectively. We can compose such matrices and $G L_{N}(m \mid n)$ is the group of invertible matrices. There is a natural projection $A \rightarrow \bar{A}$ which defines an epimorphism

$$
\pi: G L_{N}(m \mid n) \rightarrow G_{N}(m \mid n) .
$$

More generally, if $M_{N}(m \mid n)$ denotes the set of matrices of the form $A$ and $\bar{M}_{N}(m \mid n)$ the set of matrices of the form $\bar{A}$, we have a commutative diagram

$$
\begin{array}{ccc}
M_{N}(m \mid n) & \stackrel{\pi}{\rightarrow} & \bar{M}_{N}(m \mid n) \\
\varepsilon \searrow & & \swarrow \bar{\varepsilon} \\
& M(m+n) &
\end{array}
$$


where $M(m+n)$ are the $(m+n) \times(m+n)$ matrices with real entries, $\varepsilon$ is induced by the augumentation $\varepsilon: \Lambda \rightarrow R$ and $\bar{\varepsilon}$ is induced by both $\varepsilon$ and $\bar{\varepsilon}: \Lambda^{(N)} \rightarrow R$.

Proposition (2.5). The following statements are equivalent.

(1) $A$ matrix $A \in M_{N}(m \mid n)$ is invertible.

(2) The projection $\pi A \in \bar{M}_{N}(m \mid n)$ is invertible.

(3) The augumentation $\varepsilon A \in M(m+n)$ is invertible.

Thus we have a commutative diagram:

$$
\begin{array}{ccc}
G L_{N}(m \mid n) & \stackrel{\pi}{\rightarrow} & G_{N}(m \mid n) \\
\varepsilon \searrow & & \swarrow \bar{\varepsilon} \\
& G L(m, R) \times G L(n, R) &
\end{array}
$$

It is also very easy to describe $\operatorname{Ker} \pi$. Let $e^{\chi}=e^{1} \cdots e^{N}$ be a generator of $\Lambda^{N}$ and define $H$ to be the subgroup of $G L_{N}(m \mid n)$ consisting of matrices

$$
\begin{aligned}
& \left(\begin{array}{cc}
I & 0 \\
0 & I+r e^{\chi}
\end{array}\right) \text { if } N \text { is even, } \\
& \left(\begin{array}{cc}
I & r e^{\chi} \\
0 & I
\end{array}\right) \text { if } N \text { is odd, }
\end{aligned}
$$

where $r$ is a real $n \times n$ matrix if $N$ is even and an $m \times n$ matrix if $N$ is odd.

Proposition (2.8). $H$ is a normal subgroup of $G L_{N}(m \mid n)$ and $\operatorname{Ker} \pi=H$, so that $G_{N}(m \mid n)$ is the quotient of $G L_{N}(m \mid n)$ by $H$.

We now have

THEOREM (2.9). The group $G L_{N}(m \mid n)$ is homeomorphic to $G L(m, R) \times G L(n, R)$ $\times R^{t}$, where $t=2^{N-1}(m+n)^{2}-m^{2}-n^{2}$ and $H$ is homeomorphic to $R^{n^{2}}$ if $N$ is even and to $R^{m n}$ if $N$ is odd. Hence $G_{N}(m \mid n)$ is of the same homotopy type as $G L(m, R) \times$ $G L(n, R)$.

Proof. In $\Lambda$, we define $s: \Lambda \rightarrow \Lambda$ by $s(\lambda)=\lambda-\varepsilon(\lambda) \cdot 1$. This extends to matrices in $M(m \mid n)$, and thus $A=\varepsilon(A)+s(A)$ where this description is unique. If $A \in G L_{N}(m \mid n)$, then $s(A)=\left(\begin{array}{cc}s a \xi \\ \eta & s b\end{array}\right)$ runs over arbitrary matrices and counting dimensions we see the first part. Similarly, the matrices $r$ in the definition of $H$ are arbitrary, thus $H$ is homeomorphic to $R^{n^{2}}$ or $R^{m n}$ and from the fibration $H \rightarrow$ $G L_{N}(m \mid n) \stackrel{\pi}{\rightarrow} G_{N}(m \mid n)$ it follows that $\pi$ is a homotopy equivalence.

We now construct an embedding $j: G_{N}(m \mid n) \rightarrow G L\left(2^{N-1}(m+n), R\right)$ such that the following diagram commutes

$$
\begin{gathered}
G L(m, R) \times G L(n, R) \stackrel{i}{\rightarrow} G_{N}(m \mid n) \\
k \searrow j \\
G L\left(2^{N-1}(m+n), R\right)
\end{gathered}
$$


where $i$ is induced by $R \stackrel{i}{\rightarrow} \Lambda, i(t)=t 1$ and is an inclusion, and $k$ is the $2^{N-1}$-fold diagonal embedding

$$
\left(\begin{array}{ll}
a & 0 \\
0 & b
\end{array}\right) \stackrel{k}{\rightarrow}\left(\begin{array}{lllllll}
a & & & & & & 0 \\
& b & & & & & \\
& & a & & & & \\
& & & b & & & \\
& & & & \ddots & & \\
0 & & & & & a & \\
& & & & & & b
\end{array}\right) .
$$

Observe that $i$ is a homotopy equivalence.

There are two equivalent ways to define $j$. We merely outline one and describe the second fully. The matrix $\left(G_{i} f^{j}(x)\right)$ determines uniquely the Jacobian matrix of $E^{m, n} \rightarrow E^{m, n}$ which is a real $2^{N-1}(m+n) \times 2^{N-1}(m+n)$ matrix, by using $\partial f^{j} / \partial x_{\mu}^{i}$ $=e^{\mu} * G_{i} f^{j}$ and expanding further $\partial f^{j} / \partial x_{\mu}^{i}$ in terms of a basis in $\Lambda$. Using this same procedure, we obtain a function

$$
M_{N}(m / n) \rightarrow M\left(2^{N-1}(m+n)\right)
$$

which is multiplicative, $1-1$ and sends the identity to the identity and thus determines an embedding

$$
j: G_{N}(m \mid n) \rightarrow G L\left(2^{N-1}(m+n), R\right) .
$$

We now proceed to the second way. Let $V=V_{0}+V_{1}$ be a graded vector space, with $\operatorname{dim}=V_{0}=m, \operatorname{dim} V_{1}=n$ and let $U(V)$ be a free $\Lambda$-module generated by $V$. If we choose a basis $\left\{v_{i}\right\}, i=1, \ldots, m+n$, for $V$, then we can let $A \in M_{N}(m \mid n)$ act on $U(V)$ as a $\Lambda$-endomorphism by defining

$$
\begin{array}{ll}
A\left(v_{i}\right)=\sum a_{j i} v_{j}+\sum \eta_{j i} v_{j} & \text { for } 1 \leqslant i \leqslant m, \\
A\left(v_{i}\right)=\sum \xi_{j i} v_{j}+\sum b_{j i} v_{j}, & m+1 \leqslant i \leqslant m+n,
\end{array}
$$

and extend by $\Lambda$-linearity.

Since $\operatorname{dim}_{R} U(V)=2^{N}(m+n)$, the above allows us to consider $G L_{N}(m \mid n)$ as a subgroup of $G L\left(2^{N}(m+n)\right)$.

PROPOSITION (2.12). The representation of $G L_{N}(m \mid n)$ on $U(V)$ considered as a real vector space is completely reducible to representations in $U(V)_{0}$ and $U(V)_{1}$.

This easily follows from (2.11). Now the action of $G L_{N}(m+n)$ on $U(V)_{0}$ is not effective, however, we have

Proposition (2.13). The action (2.11) on $U(V)$ induces an effective action of $G_{N}(m \mid n)$ on $U(V)_{0}$ and thus it describes an embedding

$$
j: G_{N}(m \mid n) \rightarrow G L\left(2^{N-1}(m+n)\right)
$$

which makes (2.10) commutative.

Proof. Let $A$ and $A^{\prime}$ in $G L_{N}(m \mid n)$ be such that $A=A^{\prime}$ on $U(V)_{0}$. Then

$$
A\left(v_{i}\right)=A^{\prime}\left(v_{i}\right) \text { for } i=1, \ldots, m
$$


and

$$
A\left(e^{a} v_{j}\right)=A^{\prime}\left(e^{a} v_{j}\right) \text { for all } a=1, \ldots, N
$$

and $j=m+1, \ldots, m+n$. Thus $e^{a}\left(A\left(v_{j}\right)-A^{\prime}\left(v_{j}\right)\right)=0$ for all $a$ in $U(V)$. Hence

$$
A\left(v_{j}\right)-A^{\prime}\left(v_{j}\right)=e^{\chi} x_{j}
$$

since $A$ and $A^{\prime}$ preserve degree, and $v_{j} \in U(V)_{1}$, we have $e^{\chi}$ in $\Lambda_{0}$ and $x_{j} \in V_{1}$ if $N$ is even and $e^{\chi}$ in $\Lambda_{1}$ and $x_{j} \in V_{0}$ if $N$ is odd. Thus in case $N$ is even,

$$
x_{j}=\sum_{|i|=1} r_{j i} v_{i} \text { for }|j|=1 \text {. }
$$

If $N$ is odd, then

$$
x_{j}=\sum_{|i|=0} r_{j i} v_{i} \text { for }|j|=0 \text {. }
$$

Thus if $A=\left(\begin{array}{c}a \xi \\ \eta\end{array}\right)$, then

$$
A^{\prime}=\left\{\begin{array}{cc}
\left(\begin{array}{cc}
a & \xi+e^{x} r \\
\eta & b
\end{array}\right) & \text { if } N \text { odd } \\
\left(\begin{array}{cc}
a & \xi \\
\eta & b+e^{x} r
\end{array}\right) & \text { if } N \text { even }
\end{array}\right.
$$

hence

$$
A^{\prime}=\left\{\begin{array}{l}
\left(\begin{array}{cc}
1 & e^{x} r^{\prime} \\
0 & 1
\end{array}\right) A \quad \text { if } N \text { is odd } \\
\left(\begin{array}{cc}
1 & 0 \\
0 & 1+e^{x} r^{\prime}
\end{array}\right) A \quad \text { if } N \text { is even }
\end{array}\right.
$$

where $r^{\prime}=r \varepsilon(b)^{-1}$.

Finally because $\operatorname{dim}_{R} \Lambda_{0}=\operatorname{dim}_{R} \Lambda_{1}=2^{N-1}$, we may choose an order in the basis for $\Lambda$ so that the even and the odd basis elements alternate. Suppose that the basis elements are $b_{0}, b_{1}, \ldots, b_{2^{N-1}-1}$. Then a basis for $U(\Lambda)_{0}=R^{2^{N-1}(m+n)}$ is $\left\{b_{0} v_{1}, \ldots, b_{0} v_{m}, b_{1} v_{m+1}, \ldots, b_{1} v_{m+n}, b_{2} v_{1}, \ldots, b_{2} v_{m}, \ldots\right\}$. Relative to this basis $j i$ of the matrix $\left(\begin{array}{ll}\alpha & 0 \\ 0 & \beta\end{array}\right)$ is precisely $k$ of that matrix. This finishes the proof.

Theorem (2.14). Let $A \in G_{N}(m \mid n)$. Then $A$ acting on $U(V)_{0}$ satisfies

(a) $A\left(e^{\mu} \otimes v_{i}\right)=\sigma(\mu, \nu) e^{\mu-\nu} A\left(e^{\nu} \otimes v_{i}\right)$ where $|\mu|=|i|=|\nu|$ and $\nu \subset \mu$. And

(b) $e^{b} A\left(e^{a} \otimes v_{i}\right)+e^{a} A\left(e^{b} \otimes v_{i}\right)=0$ for all $i=m+1, \ldots, m+n$ and $a, b=$ $1, \ldots, N$.

Conversely if $A \in G L\left(2^{N-1}(m+n)\right)$ satisfies (a) and (b), then $A \in G_{N}(m \mid n)$.

This result is a direct consequence of the formula (i)' and (ii)" in the proof of (1.6).

3. The pseudogroups $\mathscr{S}^{1}$ and $\mathscr{S}^{\infty}$. Given manifolds $M^{a}$ and $N^{b}$, a local map $f$ : $M \rightarrow N$ is a differentiable map from an open set $U \subset M$ onto an open set $V$ of $N$. The set $U$ is called the domain of $f$, and $V$ the range of $f$. We say $f$ is a local diffeomorphism if $f: U \rightarrow V$ is a diffeomorphism. 
If $a=b=2^{N-1}(m+n)$, we say $f$ is a local $G^{1}\left(G^{\infty}\right)$ diffeomorphism if $f$ is a local diffeomorphism such that for every coordinate neighborhood $U \subset \operatorname{dom} f, f(U)$ is a coordinate neighborhood of $N$ and in the diagram

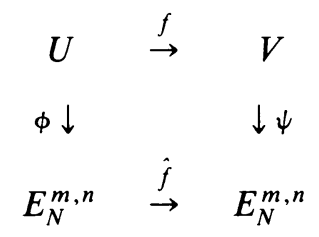

where $\hat{f}=\psi \circ f \circ \phi^{-1}$, we have $\hat{f}$ is $G^{1}\left(G^{\infty}\right)$.

We denote by $J(f)$ the Jacobian matrix of $f$.

TheOREM (3.1). A local map $f: E_{N}^{m, n} \rightarrow E_{N}^{m, n}$ is a local $G^{1}$-diffeomorphism if and only if $(J f)_{p} \in G_{N}(m \mid n)$ for all $p \in \operatorname{dom}(f)$.

Proof. This is an immediate consequence of Theorems (1.6) and (2.14).

We thus obtain

COROLlaRY (3.2). The set $\mathscr{S}^{1}$ of local $G^{1}$-diffeomorphisms of $E_{N}^{m, n}$ into itself is a pseudogroup. Moreover, $\mathscr{S}^{1}$ is precisely the pseudogroup of local diffeomorphisms whose jacobian at each point of its domain belongs to $G_{N}(m \mid n)$.

COROLlaRY (3.3). (The inverse mapping theorem.) Let $U \subset E_{N}^{m, n}$ be open and $f$ : $U \rightarrow E_{N}^{m, n}$ be $G^{1}$. Suppose also that $(J f)_{p} \neq 0$ for some $p \in U$. Then there is a neighorhood $V \subset U$ of $p$ such that $f$ restricted to $V$ is open and a bijection onto $f(V)$. Moreover $f^{-1} \mid f(V)$ is also $G^{1}$.

COROLlary (3.4). (Composite mapping theorem for local diffeorphisms.) Let $f$ and $g$ be local $G^{1}$-diffeomorphisms of $E^{m, n}$, with $\operatorname{range}(g) \subset \operatorname{dom}(f)$, then $f \circ g$ is $G^{1}$.

The more general composite mapping theorem can be obtained from Theorem (1.6).

Proposition (3.5). Suppose $E_{N}^{m, n} \stackrel{g}{\rightarrow} E_{N}^{m, n} \stackrel{f}{\rightarrow} E_{N}^{m, n}$ are $G^{1}$-maps, then $f \circ g$ is $G^{1}$ and moreover

$$
G_{i}(f \circ g)=\sum_{j=1}^{r+s}\left(G_{j} f\right) *\left(G_{i} g^{j}\right) .
$$

In order to obtain a characterization of $G^{\infty}$-diffeomorphisms similar to (3.1), it is necessary to go to higher order structures. This we do by considering higher order frame bundles; cf. [12]. actually, since the conditions are second order, it suffices to treat only second order jets.

Let $C_{0}^{\infty}\left(R^{n}, R\right)$ denote the maps of class $C^{\infty}, f: R^{n} \rightarrow R^{m}$ with $f(0)=0$. For such maps $f$, in a neighborhood of 0 , we have

$$
f^{j}(x)=\sum \frac{\partial f^{j}}{\partial x_{i}}(0) x_{i}+\sum \frac{\partial^{2} f^{j}}{\partial x_{i} \partial x_{k}}(0) x_{i} x_{k}+\cdots .
$$


The second order jet of $f, j_{2}(f)$, is the set of real numbers

$$
\left(\frac{\partial f^{j}}{\partial x_{i}}(0), \frac{\partial^{2} f^{j}}{\partial x_{i} \partial x_{k}}(0)\right) .
$$

We define an equivalence relation in $C_{0}^{\infty}\left(R^{n}, R^{n}\right)$, by setting $f \sim g$ if and only if $j_{2}(f)=j_{2}(g)$. Restricting this equivalence relation to local diffeomorphisms with 0 in their domain, we obtain a group $G(n)_{2}$ with composition as multiplication. The group $G(n)_{2}$ is a group extension of the following sort. Let $A$ be an abelian group and $G$ a group, so that $G$ acts on the left and on the right of $A$, so that if $g \cdot a$ and $a \cdot g$ denote the actions, we have $g \cdot\left(a \cdot g^{\prime}\right)=(g \cdot a) \cdot g^{\prime}$. Let $G \underline{\times} A$ denote the group which as a set is $G \times A$, and with multiplication

$$
(g, a) *\left(g^{\prime}, a\right)=\left(g g^{\prime}, g \cdot a^{\prime}+a \cdot g\right) .
$$

In the case of $G(n)_{2}$, the group $G=G L(n)$ and $A$ is the vector group isomorphic to $S^{2}(V) \otimes V^{*}$, where $V$ is an $n$-dimensional vector space, $V^{*}$ is its dual and $S^{2}(V)$ is the vector space of symmetric 2-tensors on $V$. The action of $G L(n)$ on $V$ induces naturally actions of $G L(n)$ on $S^{2}(V)$ and on $V^{*}$. If $g \in G L(n), u \otimes v$ is an element of $S^{2}(V) \otimes V^{*}, g \cdot(u \otimes v)=g \cdot u \otimes v$ and $(u \otimes v) \cdot g=u \otimes v \cdot g$ are left and right actions satisfying (3.6). Then

$$
G(n)_{2} \simeq G L(n, R) \underline{\times}\left(S^{2}(V) \otimes V^{*}\right) .
$$

Suppose now that $G$ is a Lie subgroup of $G L(n, R)$. Then we may consider the abelian group

$$
G_{1}=\operatorname{Hom}(V, \mathfrak{g}) \cap \operatorname{Hom}\left(V, S^{2}(V)\right)
$$

where $g$ is the Lie algebra of $G$.

If we restrict the left and right actions of $G L(n)$ to $G$ acting on $G_{1}$, we may form $G_{2}=G \underline{\times} G_{1}$ and $G_{2}$ is a subgroup of $G L(n)_{2}$

Suppose we consider the subgroup $G_{N}(m \mid n)$ of $G L\left(2^{N-1}(m+n), R\right)$, then by the above process we determine a subgroup $G_{N}(m \mid n)_{2}$ of $G\left(2^{N-1}(m+n)\right)_{2}$.

We now describe a subgroup of $G_{N}(m \mid n)_{2}$ that will characterize $G^{\infty}$-maps. Recall that $R^{2^{N-1}(m+n)} \simeq(\Lambda \otimes W)_{0}$, where $\Lambda$ is the exterior algebra on $R^{N}$ and $W$ is a $Z_{2}$-graded vector space, $W=W_{0}+W_{1}$ with $\operatorname{dim} W_{0}=m$, $\operatorname{dim} W_{1}=n$. Thus

$$
(\Lambda \otimes W)_{0}=\Lambda_{0} \otimes W_{0}+\Lambda_{1} \otimes W_{1} .
$$

Now we recall that $G_{N}(m \mid n)$ acts on $(\Lambda \otimes W)_{0}$ as follows. If $w_{1}, \ldots, w_{m+n}$ is an $R$-basis for $W$, and $\bar{T} \in G_{N}(m \mid n)$, lift $\bar{T}$ to $T \in G L_{N}(m \mid n)$. Then

$$
T w_{i}=\sum \alpha_{i j} \otimes w_{j}
$$

with $\left|\alpha_{i j}\right|+\left|w_{j}\right|=\left|w_{i}\right|$, and $T\left(\lambda \otimes w_{i}\right)=\lambda T\left(w_{i}\right)$. Moreover, this action is independent of the lift.

Consider now the mapping

$$
t:(\Lambda \otimes W)_{0} \otimes(\Lambda \otimes W)_{0} \rightarrow(\Lambda \otimes W)_{0} \otimes(\Lambda \otimes W)_{0}
$$


defined by

$$
t(\alpha \otimes v \otimes \beta \otimes w)= \begin{cases}\beta \otimes v \otimes \alpha \otimes w & \text { if } \alpha, \beta \in \Lambda^{1}, \\ -\alpha \otimes v \otimes \beta \otimes w & \text { otherwise. }\end{cases}
$$

Let $(\Lambda \otimes W)_{0}=V$, and restrict $t$ to $S^{2}(V)$. Finally, define $t^{*}: S^{2}(V) \otimes V^{*} \rightarrow$ $S^{2}(V) \otimes V^{*}$ by $t^{*}=t \otimes$ id.

Lemma (3.7). Let $u \in S^{2}(V) \otimes V^{*}$ and $g \in G L(V)$. If $t^{*} u+u=0$, then $t^{*}(g \cdot u)$ $+g \cdot u=0$.

Proof. If we choose a basis $\left\{e^{\mu}\right\}$ for $\Lambda$, we can write any $u \in S^{2}(V) \otimes V^{*}$ as

$$
\begin{aligned}
u= & \sum \lambda_{i, \mu, j, \nu}^{k}\left(\left(e^{\mu} \otimes w_{1}\right) \odot\left(e^{\nu} \otimes w_{j}\right)\right) \otimes v_{k}^{*} \\
& +\sum \lambda_{i, a, j, b}^{l}\left(\left(e^{a} \otimes w_{i}\right) \odot\left(e^{b} \otimes w_{j}\right)\right) \otimes v_{l}^{*},
\end{aligned}
$$

where $v_{k}^{*}, v_{l}^{*}$ are elements of $V^{*}, a \odot b$ is the symmetric tensor product of $a$ and $b$, $\lambda_{i, \mu, j, \nu}^{k}$ are real numbers with length $\mu$ or length $\nu$ greater than 1 , while $a, b$ have length 1 and

$$
\lambda_{i, \mu, j, \nu}^{k}=\lambda_{j, \nu, i, \mu}^{k}, \quad \lambda_{i, a, j, b}^{l}=\lambda_{j, b, i, a}^{l}
$$

Then

$$
t^{*} u+u=\sum\left(\lambda_{i, a, j, b}^{l}+\lambda_{i, b, j, a}^{l}\right)\left(\left(e^{a} \otimes w_{i}\right) \odot\left(e^{b} \otimes w_{j}\right)\right) \otimes v_{l}^{*} .
$$

The condition $t^{*} u+u=0$, becomes

$$
\lambda_{i, a, j, b}^{l}+\lambda_{i, b, j, a}^{l}=0 .
$$

Now look at $g \cdot u$ :

$$
\begin{aligned}
g \cdot u= & \sum \lambda_{i, \mu, j, \nu}^{k}\left(e^{\mu} g\left(w_{i}\right)\right) \odot\left(e^{\nu} g\left(w_{j}\right)\right) \otimes v_{k}^{*} \\
& +\sum \lambda_{i, a, j, b}^{l}\left(e^{a} g\left(w_{i}\right)\right) \odot\left(e^{b} g\left(w_{j}\right)\right) \otimes v_{l}^{*} .
\end{aligned}
$$

Hence

$$
\begin{aligned}
t^{*} g \cdot u+g \cdot u= & \sum \lambda_{i, a, j, b}^{l}\left(e^{b} \varepsilon(g)\left(w_{i}\right)\right) \odot\left(e^{a} \varepsilon(g)\left(w_{j}\right)\right) \otimes v_{l}^{*} \\
& +\sum \lambda_{i, a, j, b}^{l}\left(e^{a} \varepsilon(g)\left(w_{i}\right)\right) \odot\left(e^{b} \varepsilon(g)\left(w_{j}\right)\right) \otimes v_{l}^{*}
\end{aligned}
$$

and $t^{*} g \cdot u+g \cdot u=0$ follows from (3.8). Clearly

$$
t^{*} u+u=0 \text { implies } t^{*}(u \cdot g)+u \cdot g=0 \text {. }
$$

Define $S_{N}(m \mid n)_{1}=\left\{u \in G_{N}(m \mid n)_{1} \mid t^{*} u+u=0\right\}$. Then by (3.7) and (3.9), $S_{N}(m \mid n)_{1}$ is invariant under the left and right actions of $G_{N}(m \mid n)$ and is clearly a subvector space of $G_{N}(m \mid m)_{1}$. Thus we may form

$$
S_{N}(m \mid n)_{2}=G_{N}(m \mid n) \underline{x} S_{N}(m \mid n)_{1}
$$

which is a subgroup of $G_{N}(m \mid n)_{2}$.

If $f: E_{N}^{m, n} \rightarrow E_{N}^{m, n}$ is a local diffeomorphism, we define $j_{2}(f)(p)$ for $p \in \operatorname{dom}(f)$ by

$$
j_{2}(f)(p)=\left\{\frac{\partial f_{\rho}^{k}}{\partial x_{\mu}^{i}}(p), \frac{\partial^{2} f_{\rho}^{k}}{\partial x_{\mu}^{i} \partial x_{\nu}^{j}}(p)\right\}
$$


Now we have the characterization theorem for $G^{\infty}$-maps.

THEOREM (3.11). A local diffeomorphism $f: E_{N}^{m, n} \rightarrow E_{N}^{m, n}$ is $G^{\infty}$ if and only if its 2-jet $j_{2}(f)(p)$ belongs to $S_{N}(m \mid n)_{2}$ for all $p \in \operatorname{dom}(f)$.

Proof. We only show the sufficiency. If $j_{2}(f)(p) \in S_{N}(m \mid n)_{2}$ for all $p \in \operatorname{dom}(f)$, then by construction of $S_{N}(m \mid n)_{2}$, and Theorem (3.1), $f$ is $G^{1}$. Now by definition of $S_{N}(m \mid n)_{2}$, for $j_{2}(f)(p)$ as in (3.10), we have for $i$ and $j$ odd, that

$$
\frac{\partial^{2} f_{\rho}^{k}}{\partial x_{a}^{i} \partial x_{b}^{j}}(p)+\frac{\partial^{2} f_{\rho}^{k}}{\partial x_{b}^{i} \partial x_{a}^{j}}(p)=0
$$

and since this holds for all $p \in \operatorname{dom}(f)$, by Theorem (1.12), the map $f$ is $G^{\infty}$ in $\operatorname{dom}(f)$.

COROLlaRY (3.12). The set of local $G^{\infty}$-diffeomorphisms of $E_{N}^{m, n}$ into itself is a pseudogroup $\mathscr{S}^{\infty}$. Moreover $\mathscr{S}^{\infty}$ is precisely the pseudogroup of local diffeomorphisms whose 2-jet at each point of its domain belongs to $S_{N}(m \mid n)_{2}$.

This gives both the inverse and composite mapping theorems for local diffeomorphisms in the $G^{\infty}$-category. Moreover, the more general theorem holds.

Proposition (3.13). Suppose $f$ and $g$ are $G^{\infty}$-maps from $E_{N}^{m, n}$ to itself, then $f \circ g$ is also $G^{\infty}$.

As a consequence of (3.13) and (1.17), we have the following result on the $z$-expansion of a composition:

THEOREM (3.14). Let $U \subset E_{N}^{m, n}$ be open, $\tilde{f} \in C^{\infty}\left(\varepsilon(U), E_{N}^{r, s}\right)$ and $g: E_{N}^{r, s} \rightarrow E_{N}^{k, l}$ be $G^{\infty}$. Then $z(g \circ \tilde{f})=g \circ z(\tilde{f})$.

4. $G^{1}$ - and $G^{\infty}$-supermanifolds.

Definition (4.1). Let $M$ be a $C^{\infty}$-manifold. $M$ is a $G^{1}\left(G^{\infty}\right.$-)-supermanifold of type $(m, n, N)$ if $M$ has a maximal atlas, compatible with the pseudogroup $\mathscr{S}^{1}\left(\mathscr{S}^{\infty}\right)$ of local $G^{1}\left(G^{\infty}-\right)$-diffeomorphisms of $E_{N}^{m, n}$ into itself. We will also say that $M$ has an $\mathscr{S}^{1}\left(\mathscr{S}^{\infty}-\right)$-structure.

Proposition (4.2). A $G^{1}$-supermanifold of type $(m, n, N)$ is a real $C^{\infty}$-manifold of dimension $2^{N-1}(m+n)$. Moreover a $G^{\infty}$-supermanifold is a $G^{1}$-supermanifold.

Let $M, M^{\prime}$ be $G^{1}\left(G^{\infty}\right.$-)-supermanifolds of type $(m, n, N)$ and $\left(m^{\prime}, n^{\prime}, N^{\prime}\right)$ respectively. A $C^{\infty}$-map $f: M \rightarrow M^{\prime}$ is $G^{1}\left(G^{\infty}\right)$ if for any pair of allowable charts $(U, \phi)$, $\left(U^{\prime}, \phi^{\prime}\right)$ of $M$ and $M^{\prime}$ respectively with $f(U) \subset U^{\prime}$, the coordinate representative

$$
\hat{f}=\phi^{\prime} \circ f \circ \phi^{-1}: E_{N}^{m, n} \rightarrow E_{N}^{m^{\prime}, n^{\prime}}
$$

is $G^{1}\left(G^{\infty}\right)$.

We will also need the notion of a $G^{1}\left(G^{\infty}\right.$-)-map of $M$ into $\Lambda^{(k)}$. A map $f$ : $M \rightarrow \Lambda^{(k)}$ is $G^{1}\left(G^{\infty}\right)$ if for any allowable chart $(U, \phi)$ of $M$, the composite $f \circ \phi^{-1}$ is $G^{1}\left(G^{\infty}\right)$. 
Denote by $\operatorname{SJ} \hat{f}(x)$ the super Jacobian matrix of $f$ at $x$ defined by (2.1). The augumentation $\varepsilon(S J \hat{f}(x))$ is a real-valued matrix which splits into the direct sum $A+B$ of two matrices. Define the super rank $S$ rk $f(p)$ of $f$ at $p=\phi^{-1}(x) \in M$ as the pair $(k, l)$ where $k$ is the rank of $A$ and $l$ is the rank of $B . S$ rk $f(p)$ is independent of the choice of coordinate charts. We have

THEOREM (4.4). (Super rank.) Let $f: M \rightarrow M^{\prime}$ be a $G^{1}$-map of $G^{1}$-supermanifolds with constant super rank $(k, l)$. Then about every point $p \in M$ and $f(p) \in M$ there are allowable charts $(U, \phi)$ and $\left(U^{\prime}, \phi^{\prime}\right)$, respectively, such that $f(U) \subset U^{\prime}, \phi(p)=0$, $\phi^{\prime}(f(p))=0$, and $\hat{f}\left(x_{j}^{1}, \ldots, x^{m+n}\right)=\left(x^{1}, \ldots, x^{k}, 0, \ldots, 0, x^{k+1}, \ldots, x^{k+l}, 0, \ldots, 0\right)$.

The proof of this theorem is analogous to the usual theorem and will be omitted. Another standard result is

Proposition (4.5). Let $N, M, M^{\prime}$ be $G^{1}\left(G^{\infty}\right.$-)-supermanifolds. Suppose that $f$ : $M \rightarrow M^{\prime}$ is $G^{1}\left(G^{\infty}\right)$, that $i: N \rightarrow M^{\prime}$ is a $G^{1}\left(G^{\infty}-\right)$-subsupermanifold, i.e. $(i, N)$ is a submanifold with $i$ of class $G^{1}\left(G^{\infty}\right)$, and $f(M) \subset i(N)$. Then there is a map $g$ such that $g \circ i=f$. If $g$ is continuous it is $G^{1}\left(G^{\infty}\right)$.

5. Almost supermanifolds. We say a manifold $M$ of dimension $2^{N-1}(m+n)$ has an almost superstructure of type $(m, n, N)$ if the group of the tangent bundle of $M$ can be reduced to $G_{N}(m \mid n)$. By (2.10), and passing to classifying spaces we have

$$
\begin{gathered}
B G L(m, R) \times B G L(n, R) \stackrel{i}{\rightarrow} B G_{N}(m / n) \\
k \searrow \\
\quad \downarrow j \\
B G L\left(2^{N-1}(m+n), R\right)
\end{gathered}
$$

a commutative diagram and by (2.9), $i$ is a homotopy equivalence and $k$ is the diagonal embedding. We thus immediately obtain

THEOREM (5.1). The manifold $M$ of dimension $2^{N-1}(m+n)$ has an almost superstructure of type $(m, n, N)$ if and only if the tangent bundle $T(M)$ can be written as

$$
T(M)=2^{N-1}\left(\alpha_{m} \otimes \beta_{n}\right)
$$

where $\alpha_{m}$ and $\beta_{n}$ are $m$ - and $n$-plane bundles, respectively.

We also have

THEOREM (5.2). $A G^{1}$-supermanifold $M$ of type $(m, n, N)$ has an almost superstructure of type $(m, n, N)$.

Proof. This follows immediately from (3.2) and the definitions.

COROLLARY (5.3). If a manifold $M$ has an almost superstructure of type $(m, n, N)$ its Stiefel-Whitney classes $w_{i}(M)$ are zero for $i \leqslant 2^{N-1}-1$.

We now give a series of examples of almost supermanifolds. Let $R P^{k}$ be a real projective space and $S^{1}$ the unit circle. 
Proposition (5.4). The manifold $R P^{2^{N-1}(m+n)-1} \times S^{1}$ is an example of a compact manifold which admits an $(m, n, N)$-almost structure.

Proof. By (5.1), it suffices to see the splitting of the tangent bundle. But

$$
\begin{aligned}
T\left(R P^{k} \times S^{1}\right) & =\pi_{1} * T\left(R P^{k}\right)+\pi_{2} * T\left(S^{1}\right) \\
& =T\left(R P^{k}\right) \otimes 1+1 \otimes T\left(S^{1}\right)=T\left(R P^{k}\right) \otimes 1+1 \otimes 1 \\
& =\left(T\left(R P^{k}\right)+1\right) \otimes 1=(k+1)\left(\xi_{k} \otimes 1\right)
\end{aligned}
$$

where $\pi_{1}, \pi_{2}$ are the projections, $\xi_{k}$ is the Hopf line bundle over $R P^{k}$ and $\otimes$ is the exterior tensor product. Take $k=2^{N-1}(m+n)-1$, then

$$
\begin{aligned}
T\left(R P^{2^{N-1}(m+n)-1} \times S^{1}\right) & =2^{N-1}(m+n)\left(\xi_{2^{N-1}(m+n)-1} \otimes 1\right) \\
& =2^{N-1}(m \xi \otimes 1+n \xi \otimes 1)
\end{aligned}
$$

and thus (5.4) follows.

To obtain noncompact examples is much easier. Let $W$ be a manifold of dimension $m$ and $\beta$ an $n$-plane bundle over $W$. Define $T^{(k)}(W)$ inductively by

$$
T^{(k)}(W)=T\left(T^{(k-1)}(W)\right) \text { for } k \geqslant 1
$$

with $T^{0}(W)=W$ and $T^{1}(W)=T(W)$. Then

$$
T^{(k)}(W) \stackrel{p_{k}}{\rightarrow} W
$$

is a $2^{k-1} m$-plane bundle and

$$
T^{(k+1)}(W)=2^{k}\left(p_{k}^{*} T(W)\right)
$$

Thus take $k=2^{N-1}$ and let $E$ be the induced bundle $T^{(k)}(W)$ by $2^{N-1} \beta$. We have a mapping $E \stackrel{q}{\rightarrow} W$ and

Proposition (5.5). For the manifold $E$, we have

$$
T(E)=2^{N-1}\left(q^{*} T W+q^{*} \beta\right)
$$

and thus $E$ admits an almost $(m, n, N)$-superstructure.

REMARK (5.6). We will see later that certain supermanifolds $M$ of type $(m, n, N)$ determine a manifold $W$ of dimension $m$ and an $n$-plane bundle $\beta$ over $W$ and a map $q: M \rightarrow W$ so that

$$
T(M)=2^{N-1}\left(q^{*} T W \otimes q^{*} \beta\right)
$$

except that in most cases $q$ is not a fibration, but just a submersion.

6. The tangent and supertangent bundles. On a $G^{\infty}$-manifold $M$, we denote by $G^{\infty}(p)$ the $Z_{2}$-algebra of germs of $G^{\infty}$-functions at $p \in M$ and by $C^{\infty}(p, \Lambda)=$ $C^{\infty}(p) \otimes \Lambda$ the algebra of germs of $C^{\infty}$-functions into $\Lambda$.

If $A$ and $B$ are graded $Z_{2}$-algebras, a map $f: A \rightarrow B$ is homogeneous of degree 0 if $f\left(A_{i}\right) \subset B_{i}$, and homogeneous of degree 1 if $f\left(A_{0}\right) \subset B_{1}, f\left(A_{1}\right) \subset B_{0}$. We denote by $|f|$ the degree of $f$.

Consider a homogeneous map $F: G^{\infty}(p) \rightarrow \Lambda^{(k)}$. 
Let $\varepsilon: \Lambda^{(k)} \rightarrow R$ be the augmentation. Then we define

$$
|\varepsilon \circ F|=\left\{\begin{array}{cc}
0 & \text { if } \varepsilon F=0 \\
|F| & \text { if } \varepsilon F \neq 0
\end{array}\right.
$$

A homogeneous graded derivation $D$ is a homogeneous map $D: G^{\infty}(p) \rightarrow$ $\Lambda^{(N+1)-|\varepsilon \circ D|)}$ satisfying

$$
\begin{gathered}
D(\alpha f+\beta g)=(-1)^{|D||\alpha|} \alpha D f+(-1)^{|D||\beta|} \beta D g, \\
D(f g)=(D f) g(p)+(-1)^{|D||f|} f(p) D g
\end{gathered}
$$

where $\alpha, \beta$ are homogeneous elements in $\Lambda, f$ is a homogeneous element of $G^{\infty}(p)$ and $\|$ is the degree.

We make the set $S T_{p}(M)$ of graded derivations at $p$ into a $\Lambda$-module, by defining

$$
(\alpha * D)(f)=\alpha *(D f)
$$

for any $\alpha \in \Lambda, D \in S T_{p}(M)$ and any $f \in G^{\infty}(p)$.

The next proposition describes the structure of $S T_{p}(M)$, the supertangent space of $M$ at $p$ as a $\Lambda$-module.

Proposition (6.4). If $M$ is a $G^{\infty}$-manifold of type $(m, n, N)$, then at every point $p$ of $M, S T_{p}(M)$ is a free $\Lambda$-module on a $Z_{2}$-graded real vector space $V=V_{0}+V_{1}$ with $\operatorname{dim} V_{0}=m, \operatorname{dim} V_{1}=n$.

Proof. Since the result is local, we may suppose that $M=E_{N}^{m, n}$ and that $U$ is a neighborhood of the point $p$. The derivations $G_{i}$ of $(1.3)$ restrict to $G^{\infty}(p)$ to produce derivations $D_{i}, 1 \leqslant i \leqslant m+n$. We now check that the $D_{i}$ generate $S T_{p}(M)$ as a $\Lambda$-module. If $f \in G^{\infty}(p)$ then we may write

$$
f(x)=f(p)+\sum\left(x^{i}-p^{i}\right) * h_{i}(x)
$$

with $h_{i}(x) \in G^{\infty}(p)$ for $1 \leqslant i \leqslant m$ and $h_{i}(x) \in G^{\infty}\left(p, \Lambda^{(N)}\right)$ for $m+1 \leqslant i \leqslant m$ $+n$. Then $h_{i}(p)=D_{i} f$ and

$$
D f=\sum D\left(x^{i}\right) * D_{i} f
$$

which shows that the $D_{i}$ generate $S T_{p}(M)$.

We show that the $D_{i}$ are linearly independent over $\Lambda$. Suppose $\sum \lambda^{i} * D_{i}=0$. Then taking $x^{i}$, we find $\lambda^{i} * D_{i}\left(x^{i}\right)=0$, so $\lambda^{i} *(1)=0$, where $1 \in \Lambda$ if $|i|=0$ and then $\lambda^{i}=0$, or $1 \in \Lambda^{(N)}$ if $|i|=1$ and then both $\varepsilon\left(\lambda^{i}\right)=0$ and $s\left(\lambda^{i}\right)=0$, by the pairing (1.1). Thus (6.3) follows.

REMARK (6.5). It was crucial to use the pairing * in the module structure (6.3) to make $S T_{p}(M)$ a free $\Lambda$-module.

We now define the supertangent bundle on a $G^{\infty}$-manifold $M$ by

$$
S T M=\bigcup_{p \in M} S T_{p} M
$$

It is a vector bundle with fiber dimension $2^{N}(m+n)$. Moreover, we have 
THEOREM (6.7). If $M$ is a $G^{\infty}$-manifold, then $S T M$ has $G L_{N}(m \mid n)$ as group of the bundle. Moreover $(S T M)_{0} \simeq T M$, with group of the bundle $G_{N}(m \mid n)$ and both $T M$ and $S T M$ are $G^{\infty}$-manifolds and the natural projections to $M$ are $G^{\infty}$-maps.

If $U \subset M$ is an open set, we let $\mathscr{D}(U)$ denote the set of $C^{\infty}$-graded derivations in $U$, i.e. an element $D \in \mathscr{D}(U)$ is a mapping: $D: C^{\infty}(U, \Lambda) \rightarrow C^{\infty}(U, \Lambda)$ satisfying (6.1) and (6.2). If $M$ is a supermanifold, we let $\mathscr{D}^{\infty}(U)$ denote the set of $G^{\infty}$-graded derivations on $U$, i.e. mappings

$$
D: G^{\infty}\left(U, \Lambda^{(k)}\right) \rightarrow G^{\infty}\left(U, \Lambda^{(k-|\varepsilon \circ D|)}\right)
$$

satisfying (6.1) and (6.2). If $(U, x)$ is a local $\mathscr{S}^{\infty}$ coordinate neighborhood, then as above

$$
D f=\sum D x^{i} * G_{i} f .
$$

From (6.8) one has immediately

Proposition (6.9). $D \in \mathscr{D}(U)$ is in $\mathscr{D}^{\infty}(U)$ if and only if $D\left(x^{i}\right) \in G^{\infty}(U)$.

As usual, $D \in \mathscr{D}(U)$ is a local $C^{\infty}$-section of $S T(M)$ and $D \in \mathscr{D}^{\infty}(U)$ is a local $G^{\infty}$-section of $S T(M)$. The local $G^{\infty}$-sections on $S T(M)$ form a presheaf and we denote by $\mathscr{S} T(M)$ the sheaf of germs of $G^{\infty}$-sections of $S T(M)$. A (local) $G^{\infty}$-section $X$ is called a $G$-supervectorfield.

We finish this section with two propositions which amount to the $G^{\infty}$-analogue of the existence and uniquess theorem for systems of ordinary differential equations. (A similar result using a different sheaf of functions has been proved [16] for Berezin's approach.) This is phrased in terms of local 1-parameter supergroups. For $t \in \Lambda_{\alpha}$, $\alpha=0,1$, we denote by $G$ the superderivative with respect to $t$. If $I_{\alpha} \subset \Lambda_{\alpha}$ is an open set containing 0 and $M$ is a $G^{\infty}$-manifold, a $G^{\infty}$-map $c: I_{\alpha} \rightarrow M$ is called an even $G^{\infty}$-curve if $\alpha=0$ and an odd $G^{\infty}$-curve if $\alpha=1$. Evidently, from (1.21) we have

LEMMA (6.10). Let $c$ be an odd $G^{\infty}$-curve and $(U, x)$ an $\mathscr{S}^{\infty}$-chart in $M$, then there are $\alpha^{i} \in \Lambda_{|i|}^{(N)}$ and $\beta^{i} \in \Lambda_{|i|}$ such that

$$
\left(x^{i} \circ c\right)(t)=\alpha^{i} * t+\beta^{i} .
$$

Let $\phi_{t}: M \rightarrow M$ be a family of local $G^{\infty}$-diffeomorphisms. We call $\phi_{t}$ a local 1-parameter supergroup of transformations if the map $\phi: I_{\alpha} \times M \rightarrow M$ defined by $\phi(t, p)=\phi_{t}(p)$ satisfies:

(1) $\phi$ is $G^{\infty}$ in $t$,

(2) $\phi_{t} \circ \phi_{s}=\phi_{t+s}$ whenever $s, t, t+s \in I_{\alpha}$,

(3) $\phi_{0}=$ identity.

Proposition (6.11). Let $X$ be an odd $G^{\infty}$-supervectorfield on $M$. Then every point $p \in M$ has an $\mathscr{S}^{\infty}$-chart $(U, x)$ and a unique $G^{\infty}$ odd curve $(c(t))$ such that in $U$,

$$
G\left(x^{i} \circ c\right)(t)=(-1)^{|i|+1} X\left(x^{i}\right)(c(0)) \text {. }
$$

Furthermore, $c(t)$ defines a 1-parameter supergroup $\phi_{t}$ if and only if $X^{2}=0$.

Proof. We define for $p \in U \subset M$, a curve $c$ by

$$
\left(x^{i} \circ c\right)(t)=\left(X\left(x^{i}\right)(p)\right) * t+x^{i}(p) .
$$


Then clearly (6.12) is satisfied. Now for any $f \in G^{\infty}(U), f \circ c: I \rightarrow \Lambda$ is $G^{\infty}$ and hence by $(1.21)$ is linear, thus $(f \circ c)(t)=(X(f)(c(0))) * t+f(c(0))$.

Consider now $\phi_{t}: M \rightarrow M$ defined by $\phi_{t}(p)=c(t)$, where $c$ is the unique curve (6.13). Then

$$
\begin{aligned}
\left(x^{i} \circ \phi_{t} \circ \phi_{s}(p)\right) & =\left(x^{i} \circ \phi_{t}\right)(c(s))=\left(x^{i} \circ \hat{c}\right)(c(s)) \\
& =X\left(x^{i}\right)(c(s)) * t+x^{i}(c(s)) \\
& =X\left(x^{i}\right)(c(s)) * t+X\left(x^{i}\right)(p) * s+x^{i}(p) .
\end{aligned}
$$

Now consider the function $X\left(x^{i}\right) \circ c: I \rightarrow \Lambda$, by the above $\left(X\left(x^{i}\right) \circ c\right)(s)=$ $X^{2}\left(x^{i}\right) c(0) * s+X\left(x^{i}\right)(p)$. Hence

$$
x^{i} \circ \phi_{t} \circ \phi_{s}(p)=\left(X^{2}\left(x^{i}\right)(p) * s t+\left(X\left(x^{i}\right)(p)\right) *(t+s)+x^{i}(p)\right)
$$

and thus

$$
x^{i} \circ \phi_{t} \circ \phi_{s}(p)=x^{i} \circ \phi_{t+s}(p) \text { for all } p,
$$

and is a 1-parameter supergroup, if and only if $X^{2}=0$.

Finally, for even curves we have

Proposition (6.14). Let $X$ be an even $G^{\infty}$-supervectorfield on $M$. Then for every $p \in M$, there are neighborhoods $U$ of $p, I_{0}$ of 0 in $\Lambda_{0}$, an allowable chart $(U, x)$ and $a$ unique local 1-parameter supergroup $\phi_{t}$ satisfying

$$
G\left(x^{i} \circ c\right)(t)=X\left(x^{i} \circ \phi_{t}\right)
$$

where $c$ is an integral curve of $X$, with $c(0)=p$.

Proof. By (6.7) $S T(M)_{0} \cong T(M)$, and we may write $X$ in local coordinates as

$$
X \mid U=\sum_{|i|+|\mu| \text { even }} X_{\mu}^{i} \partial_{x_{\mu}^{i}} .
$$

Then by the standard theorem for differentiable manifolds, there exists a local 1-parameter group $\hat{\phi}_{t_{0}}$ which in local coordinates for $M$ as a manifold satisfies

$$
d\left(x_{\mu}^{j} \circ \hat{\phi}_{t_{0}}\right) / d t_{0}=X\left(x_{\mu}^{i} \circ \hat{\phi}_{t_{0}}\right) .
$$

Furthermore, there are neighborhoods $U$ of $p \in M$ and $J$ of $0 \in R$ such that $\hat{\phi}$ : $J \times U \rightarrow U$ is $C^{\infty}$ and $\hat{\phi}_{t}: U \rightarrow U$ is a $C^{\infty}$-diffeomorphism for all $t_{0} \in J$. Let $x^{i}$ denote the supercoordinates $x^{i}=\sum x_{\mu}^{i} e^{\mu}$, where the sum is taken over all $i$ and $\mu$ with $|i|+|\mu|$ even. There is a neighborhood $I_{0}$ of $0 \in \Lambda_{0}, \varepsilon\left(I_{0}\right) \subset J$, such that fixing $p \in U, x^{i} \circ \hat{\phi}(p, t) \in C^{\infty}\left(\varepsilon\left(I_{0}\right), \Lambda\right)$. By (1.16) and (1.17), there is a unique $\phi=z(\hat{\phi})$ $\in G^{\infty}\left(I_{0}\right)$ such that $\left.\phi\right|_{\varepsilon\left(I_{0}\right)}=\hat{\phi}$. Furthermore, by (3.14) and (6.15), $G z\left(x^{i} \circ \hat{\phi}\right)=$ $z\left(X\left(x^{i} \circ \hat{\phi}\right)\right)$. Since $X$ is $G^{\infty}$ and $x^{i} \circ \phi$ is $G^{\infty}, X\left(x^{i} \circ \phi\right)$ is $G^{\infty}$ and $z\left(X\left(x^{i} \circ \phi\right)\right)=$ $X\left(x^{i} \circ \phi\right)$. Also $z\left(x^{i} \circ \hat{\phi}\right)=x^{i} \circ \phi$ and (6.14) follows. The uniqueness of $\phi$ implies $\phi_{t}$ is a local 1-parameter supergroup.

7. Multifoliations. Let $M$ be an almost supermanifold, then the tangent bundle $T(M)$ has the structure of the even part of a bundle of free $\Lambda$-modules. More precisely let $U \subset M$ be open and

$$
X_{i}: U \rightarrow E(\alpha), \quad i=1, \ldots, m,
$$


$m$ linearly independent local sections of the vector bundle $\alpha$ of Theorem (5.1). Similarly let

$$
X_{m+j}: U \rightarrow E(\beta), \quad j=1, \ldots, n,
$$

be $n$ linearly independent sections of $\beta$. Then $\left(X_{1}, \ldots, X_{m+n}\right)$ is a local moving frame for $E(\alpha \oplus \beta)$. Furthermore, at each $p \in U, T_{p}(M)$ has the structure of the even part of a free $\Lambda$-module. It follows that $X_{i}^{\mu}=X_{i} \otimes e^{\mu},|i|+|\mu|=0$, is a basis of $T_{p}(M)$ for every $p \in U$. Moreover, $T_{p}(M)$ is a free $\Lambda_{0}$-module and the $\Lambda_{0}$ action on the basis $\left\{X_{i}^{\mu}\right\}$ implies for $\nu$ even and all $\mu$

$$
X_{i}^{\mu+\nu}=\sigma(\mu+\nu, \nu) X_{i}^{\mu} e^{\nu} .
$$

Now if $I\left(e^{\mu}\right)$ is the principal ideal generated by $e^{\mu} \in \Lambda$ then let $I\left(e^{\mu}\right)_{0}$ and $I\left(e^{\mu}\right)_{1}$ denote the even and odd parts respectively. On $U, E^{\mu}$ defined as

$$
\left(E(\alpha) I\left(e^{\mu}\right)_{0}\right) \oplus\left(E(\beta) I\left(e^{\mu}\right)_{1}\right)
$$

has a basis $\left\{X_{i}^{\lambda}, \lambda \supset \mu\right\}$. But it is readily checked that $E^{\mu}$ is invariant under the $G_{N}(m \mid n)$ action on $\left.T(M)\right|_{U}$. Thus for almost supermanifolds we have

Proposition (7.2). For every $\mu, E^{\mu}=\left(E(\alpha) I\left(e^{\mu}\right)_{0}\right) \oplus\left(E(\beta) I\left(e^{\mu}\right)_{1}\right)$ is a subbundle of $T(M)$ of fibre dimension $2^{N-1-l(\mu)}(m+n)$.

Proof. We need only check the dimensions. We have $I\left(e^{\mu}\right) \cong e^{\mu} \cdot \Lambda(W)$, where $W$ is a vector space of dimension $N-l(\mu)$. So $\operatorname{dim} I\left(e^{\mu}\right)=2^{N-l(\mu)}$ and hence $\operatorname{dim} I\left(e^{\mu}\right)_{0}=\operatorname{dim} I\left(e^{\mu}\right)_{1}=2^{N-l(\mu)}-1$.

As a corollary of this result and Theorem (5.1) we have

COROllaRY (7.3). If $l(\mu)=l(\nu)$, then $E^{\mu} \simeq E^{\nu}$ canonically.

The main result of this section is

TheOREM (7.4). Let $M$ be a $G^{1}$-supermanifold. Then for every $\mu \neq 0 \in S, E^{\mu}$ is an integrable subbundle. If $l(\mu) \geqslant 2$ the leaves of the corresponding foliation are all flat affine manifolds. Furthermore, if $M$ is a $G^{\infty}$-manifold, then all the leaves of all the integrable subbundles $E^{\mu}$ are flat affine manifolds.

We need the following lemma whose proof follows directly from (1.6) and will be omitted.

LeMma (7.5). Let $U \subset E_{N}^{m, n}$ be open, $f \in C^{1}\left(U, \Lambda^{(k)}\right)$, and write $f=\sum_{\mu} f_{\mu} e^{\mu}$. Then $f \in G^{1}\left(U, \Lambda^{(k)}\right)$ if and only if for every $\mu \in S, f_{\mu}$ satisfies

$$
\begin{gathered}
\frac{\partial f_{\mu}}{\partial x_{\nu}^{i}}=0 \quad \text { if } \nu \not \subset \mu . \\
\frac{\partial f_{\mu}}{\partial x_{\nu}^{i}}=\sigma(\nu, \rho) \sigma(\mu, \nu-\rho) \frac{\partial f_{\mu-(\nu-\rho)}}{\partial x_{\rho}^{i}}
\end{gathered}
$$

for all $\rho \subset \nu \subset \mu$, and $|\nu-\rho|=0$. 


$$
\frac{\partial f_{\nu-b}}{\partial x_{a}^{i}}=-\sigma(\nu, a) \sigma(\nu, b) \frac{\partial f_{\nu-a}}{\partial x_{b}^{i}}
$$

for all $a \neq b$ and $a, b \in \nu$.

Proof of Theorem (7.4). To prove the first statement we consider the underlying almost superstructure on $M$ determined in a local coordinate chart $(U, x)$ by $\left\{\partial_{x_{\mu}^{i}}=e^{\mu} \otimes G_{i}\right\}$.

Now let $\left(U^{\prime}, x^{\prime}\right)$ be another coordinate chart compatible with the pseudogroup $\mathscr{S}^{1}$. Then by (1) of (7.5) the transition functions $x^{\prime} \circ x^{-1}$ satisfy $\partial x_{\lambda}^{\prime j} / \partial x_{\mu}^{j}=0$ unless $\mu \subset \lambda$. It follows that $\left\{\partial_{x_{\nu}^{i}}: \nu \supset \mu\right\}$ extends to global commuting sections of $E^{\mu}$. Thus $E^{\mu}$ is integrable.

To prove the second statement of the theorem, we must show that the pseudogroup $\mathscr{S}^{1}$ restricted to any leaf $\mathscr{L}$ of the foliation $E^{\mu}$ with $l(\mu) \geqslant 2$ is contained in the affine pseudogroup on $\mathscr{L}$. That is, we must show that for every $\nu, \rho, \sigma \in S$ containing $\mu$ a change of coordinates $\mathscr{S}^{1}$ satisfies $\partial^{2} x_{\nu}^{\prime k} / \partial x_{\rho}^{i} \partial x_{\sigma}^{j}=0$ for all $i, j$, $k=1, \ldots, m+n$. Suppose $\rho$ is odd, then necessarily $l(\rho)>1$ and by (2) of (7.5)

$$
\frac{\partial^{2} x_{\nu}^{\prime k}}{\partial x_{\sigma}^{i} \partial x_{\rho}^{i}}=\sigma(\rho, a) \sigma(\nu, \rho) \frac{\partial^{2} x_{\nu-(\rho-a)}^{\prime k}}{\partial x_{\sigma}^{i} \partial x_{a}^{j}}
$$

for each $a \in \rho$. The result will follow from (1) of (7.5) if we show that $\sigma \not \subset \nu-(\rho-$ $a$ ). Again by (1) of (7.5) it suffices to restrict ourselves to those $\sigma, \rho$ contained in $\nu$. But by hypothesis $\mu \subset \rho, \sigma, \nu$, so it suffices to show that $\mu \not \subset \nu-(\rho-a)$. Since $\rho \supset \mu$ the only way $\nu-(\rho-a)$ can contain $\mu$ is if $\rho=a$. But this contradicts $l(\rho) \geqslant l(\mu)>1$. The case $\rho$ even is proved similarly.

To prove the last statement we argue as above to reduce our considerations to showing that $\partial^{2} x_{v}^{\prime k} / \partial x_{a}^{i} \partial x_{a}^{j}=0$. But this clearly follows from Theorem (1.12). This completes the proof of the theorem.

REMARK. By (1.10) the hypothesis that $M$ be $G^{\infty}$ in the last statement of the theorem is needed only for $\nu=\chi=1 \cdots N$ in the formula above.

Let us look more closely at the foliation involved. For any $\mu=\mu_{0}$ consider a flag of sequences $\mu_{0} \subset \mu_{1} \subset \cdots \subset \mu_{k}=\chi$ where the length $l$ satisfies $l\left(\mu_{i+1}\right)=l\left(\mu_{i}\right)+$ 1. This gives rise to a flag of subbundles

$$
E^{\chi} \subset \cdots \subset E^{\mu_{0}} \subset T(M) .
$$

Moreover, we have just seen that each subbundle is integrable, and if $E^{\nu} \subset E^{\mu}$ then each leaf of $E^{\nu}$ is contained in a leaf of $E^{\mu}$. Hence, if we consider the set $\mathscr{T}=\left\{E^{\mu} \subset T(M): \mu \in S\right\}$, then $\mathscr{T}$ has a natural partial ordering. Let $E^{\mu}, E^{\nu} \in \mathscr{T}$, we say that $E^{\mu}$ meets $E^{\nu}$, written $E^{\mu} \wedge E^{\nu}$, if there is an $E^{\lambda}$ in $\mathscr{T}$ such that every leaf of $E^{\lambda}$ is contained in both a leaf of $E^{\mu}$ and a leaf of $E^{\nu}$ and if $E^{\sigma} \in \mathscr{T}$ with the same property then $E^{\sigma} \subset E^{\lambda}$. Similarly, we say that $E^{\mu}$ joins $E^{\nu}$, written $E^{\mu} \vee E^{\nu}$, if there is an $E^{\lambda} \in \mathscr{T}$ such that $E^{\mu} \subset E^{\lambda}, E^{\nu} \subset E^{\lambda}$ and for any other $E^{\sigma}$ having this property, $E^{\lambda} \subset E^{\sigma}$. We see that $E^{\mu} \vee E^{\nu}=E^{\mu \cap \nu}$ and $E^{\mu} \wedge E^{\nu}=E^{\mu+\nu}$. Thus $\mathscr{T}$ is a lattice. We call $\mathscr{T}$ a lattice of foliations on $M$. Since for any two subbundles $E^{\mu}$ and 
$E^{\nu}, E^{\mu} \vee E^{\nu} \subset E^{0}=T(M)$ and $E^{\mu} \wedge E^{\nu} \supset E^{\chi}, \mathscr{T}$ is complete. Furthermore, $E^{\mu} \wedge$ $\left(E^{\nu} \vee E^{\lambda}\right)=\left(E^{\mu} \wedge E^{\nu}\right) \vee\left(E^{\mu} \wedge E^{\lambda}\right)$, and $E^{\mu} \vee\left(E^{\nu} \wedge E^{\lambda}\right)=\left(E^{\mu} \vee E^{\lambda}\right)$, so we have

COROllaRY (7.6). For every $G^{1}$-supermanifold $M$, the set $\mathscr{T}$ defines a complete distributive lattice of foliations on $M$.

Actually there are more foliations than those in the lattice $\mathscr{T}$. Let $E^{\mu}, E^{\nu} \in \mathscr{T}$ and denote by $E^{\mu}+E^{\nu}$ the subbundle obtained by taking the vector space sum (not direct) at each point $p \in M$. This is the space generated by the ideal $I\left(e^{\mu}, e^{\nu}\right)$, i.e. $\left(V \otimes I\left(e^{\mu}, e^{\nu}\right)_{0}\right)$, and is thus integrable since it is invariant under $\mathscr{S}^{1}$. We have

Proposition (7.7). $E^{\mu}+E^{\nu}$ is integrable.

In particular, define

$$
E=\sum_{a=1}^{N} E^{a} .
$$

$E$ is generated by the augmentation ideal $I(\Lambda)$. We will refer to $E$ as the augmentation bundle or augmentation foliation. Its fibre codimension is $m$. Let $Q=T(M) / E$ denote the normal bundle and $\operatorname{Pont}(Q)$ the Pontryagin ring of $Q$. Then by Bott's vanishing theorem

Corollary (7.8). If $M$ is $a G^{1}$-supermanifold, then $\operatorname{Pont}^{q}(Q)=0$ for all $q>2 m$.

Denote by $M_{0}$ the space of leaves of the augmentation foliation and let $\rho$ : $M \rightarrow M_{0}$ denote the projection map. It is not always possible to give $M_{0}$ the structure of a $C^{\infty}$-manifold which makes $\rho$ a $C^{\infty}$-map. In fact it is well known [13] that this is possible if and only if the foliation is regular, i.e. if $(U, x)$ is a chart of $\mathscr{S}^{1}$ (or $\mathscr{S}^{\infty}$ ) then every leaf intersects $U$ in only one slice. We will say that $M$ is a regular $G^{1}$ - or $G^{\infty}$-supermanifold if the augmentation foliation $E$ is regular. In this case $M_{0}$ is a $C^{\infty}$-manifold (not necessarily Hausdorff) and $\rho: M \rightarrow M_{0}$ is a submersion. Furthermore, every leaf $\rho^{-1}(p), p \in M_{0}$, is a closed submanifold of $M$.

To see that not all $G^{\infty}$-supermanifolds are regular, consider any $G^{\infty}$-supermanifold of type $(m, n ; 1)$. This is equivalent to giving a manifold of dimension $(m+n)$ with a foliation of codimension $m$ all of whose leaves are flat affine. As a counterexample to regularity consider the torus $S^{1} \times S^{1}$ with the foliation determined by an irrational flow. This gives $S^{1} \times S^{1}$ a $G^{\infty}$-structure of type $(1,1 ; 1)$ which is clearly not regular.

\section{REFERENCES}

1. M. Batchelor, The structure of supermanifolds, Trans. Amer. Math. Soc. 253 (1979), 329-338.

2. __ Two approaches to supermanifolds, Trans. Amer. Math. Soc. 258 (1980), 257-270.

3. F. A. Berezin, The method of second quantization, Academic Press, New York, 1966.

4. F. A. Berezin and G. I. Kac, Lie groups with commuting and anticommuting parameters, Math. USSR-Sb. 11 (1970), 311-326.

5. F. A. Berezin and D. A. Leites, Supermanifolds, Soviet Math. Dokl. 16 (1975), 1218-1222.

6. C. P. Boyer and S. Gitler, The theory of $G^{\infty}$-supermanifolds, Group Theoretical Methods in Physics (K. B. Wolf., Ed.), Lecture Notes in Physics, vol. 135, Springer-Verlag, New York 1980, pp. 532-536. 
7. C. P. Boyer, On the structure of supermanifolds, Symposium on Algebraic Topology in Honor of José Adem (S. Gitler, Ed.), Contemp. Math., vol. 12, Amer. Math. Soc., Providence, R. I., 1982, pp. 53-59.

8. B. DeWitt, Differential supergeometry (unpublished manuscript).

9. K. Kodaira and D. C. Spencer, Multifoliate structures, Ann. of Math. (2) 74 (1961), 52-100.

10. B. Kostant, Graded manifolds, graded Lie theory and prequantization, Differential Geometric Methods in Physics, Lecture Notes in Math., vol. 570, Springer-Verlag, New York, 1977, pp. 177-300.

11. D. A. Leites, Introduction to the theory of supermanifolds, Russian Math, Surveys 35 (1980), 1-64.

12. P. Molino, Théorie des G-structures: Le probleme d'equivalence, Lecture Notes in Math., vol. 588, Springer-Verlag, New York, 1977.

13. G. Reeb, Sur certaines propriétés topologiques des variétés feuilletées, Actualités Sci. Indust., no. 11, Hermann, Paris, 1952, pp. 93-158.

14. V. Rittenberg and M. Scheunert, Elementary constructions of graded Lie groups, J. Math. Phys. 19 (1978), 709-713.

15. A. Rogers, A global theory of supermanifolds, J. Math. Phys. 21 (1980), 1352-1365.

16. V. N. Shanker, Vector fields and differential equations supermanifolds, Funktsional Anal. i Prilozhen 14 (1980), 91-92.

InSTituto de InVestigaciones en Matemáticas Aplicadas y Sistemas, Universidad NaCional Autónoma de México, MÉxico 20, D. F.

Departamento de Matemáticas, Centro de Investigación y de Estudios Avanzados del IPN, MÉxico 14, D. F. (Current address of Samuel Gitler)

Current address (C. P. Boyer): Department of Mathematics and Computer Science, Clarkson College, Potsdam, New York 13676 